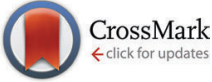

Cite this: Phys. Chem. Chem. Phys., 2016, 18, 29756

Received 2nd September 2016 Accepted 30th September 2016

DOI: $10.1039 / c 6 c p 06071 d$

www.rsc.org/pccp

\section{Deprotonation of formic acid in collisions with a liquid water surface studied by molecular dynamics and metadynamics simulations $\dagger$}

\author{
Garold Murdachaew, ${ }^{* a}$ Gilbert M. Nathanson, ${ }^{b}$ R. Benny Gerber ${ }^{\text {acd }}$ and \\ Lauri Halonen ${ }^{a}$
}

\begin{abstract}
Deprotonation of organic acids at aqueous surfaces has important implications in atmospheric chemistry and other disciplines, yet it is not well-characterized or understood. This article explores the interactions of formic acid (FA), including ionization, in collisions at the air-water interface. Ab initio molecular dynamics simulations with dispersion-corrected density functional theory were used. The 8-50 picosecond duration trajectories all resulted in the adsorption of FA within the interfacial region, with no scattering, absorption into the bulk or desorption into the vapor. Despite the known weak acidity of FA, spontaneous deprotonation of the acid was observed at the interface on a broad picosecond timescale, ranging from a few picoseconds typical for stronger acids to tens of picoseconds. Deprotonation occurred in $4 \%$ of the trajectories, and was followed by Grotthuss proton transfer through adjacent water molecules. Both sequential and ultrafast concerted proton transfer were observed. The formation of contact ion pairs and solvent-separated ion pairs, and finally the reformation of neutral FA, both trans and cis conformers, occurred in different stages of the dynamics. To better understand the deprotonation mechanisms at the interface compared with the process in bulk water, we used well-tempered metadynamics to obtain deprotonation free energy profiles. While in bulk water FA deprotonation has a free energy barrier of $14.8 \mathrm{~kJ} \mathrm{~mol}{ }^{-1}$, in fair agreement with the earlier work, the barrier at the interface is only $7.5 \mathrm{~kJ} \mathrm{~mol}^{-1}$. Thus, at the air-water interface, FA may dissociate more rapidly than in the bulk. This finding can be rationalized with reference to the dissimilar aqueous solvation and hydrogen-bonding environments in the interface compared to those in bulk liquid water.
\end{abstract}

\section{Introduction}

Acid deprotonation and proton transfer (PT) at aqueous interfaces is a fundamental chemical reaction important in many fields. The interface is most often air-water ("soft") or a wetted icy or mineral interface ("hard"). ${ }^{1}$ In particular, the protonation state of common strong acids $\left(\mathrm{HCl}\right.$ and $\left.\mathrm{HNO}_{3}\right)$ at airaqueous interfaces has been studied both experimentally, ${ }^{2-4}$ and theoretically. ${ }^{1,3-8}$ Particular interest in these studies has been determining if the acid's strength and deprotonation mechanisms and timescales at aqueous interfaces differ compared

\footnotetext{
${ }^{a}$ Laboratory of Physical Chemistry, Department of Chemistry, P. O. Box 55 (A.I. Virtasen aukio 1), FI-00014 University of Helsinki, Finland. E-mail: garold.murdachaew@helsinki.fi

${ }^{b}$ Department of Chemistry, University of Wisconsin-Madison, Madison, Wisconsin 53706, USA

${ }^{c}$ Institute of Chemistry and the Fritz Haber Research Center, The Hebrew University, 91904 Jerusalem, Israel

${ }^{d}$ Department of Chemistry, University of California, Irvine, California 92697, USA

$\dagger$ Electronic supplementary information (ESI) available. See DOI: 10.1039/c6cp06071d
}

to their known behavior in bulk ambient liquid water. $\mathrm{HNO}_{3}$, while a strong acid in liquid water, was found to remain mainly in its neutral form at the air-water interface (also depending on its concentration). ${ }^{1-7}$ With regard to $\mathrm{HCl}$, just as in the bulk, deprotonation occurs at the interface. However, while in the bulk PT quickly leads to a solvent-separated ion pair (SSIP) of $\mathrm{Cl}^{-} \cdots\left(\mathrm{H}_{2} \mathrm{O}\right)_{n} \cdots \mathrm{H}_{3} \mathrm{O}^{+}, n \geq 1$, recently, Baer et al. ${ }^{1}$ have shown that at the interface the contact ion pair (CIP) of $\mathrm{Cl}^{-} \cdots \mathrm{H}_{3} \mathrm{O}^{+}$is uniquely stable. Therefore, the simple impression of the higher concentration of under-coordinated water molecules at the interface leading to enhanced reactivity need not always be true.

Our own recent theoretical studies have examined the behavior of $\mathrm{HCl}$ and $\mathrm{H}_{2} \mathrm{SO}_{4}$ at hard interfaces: the air-wetted hydroxylated quartz interface ${ }^{9,10}$ and $\mathrm{HNO}_{3}$ at the air-ice interface including defective ice and ice with a quasi-liquid layer. ${ }^{11-13}$ It has been suggested that while ideal ice surfaces may significantly reduce deprotonation of such strong acids, real ice surfaces at temperatures greater than about $250 \mathrm{~K}$ will allow deprotonation due to the presence of the apparently ubiquitous quasi-liquid layer or defects, ${ }^{11-13}$ while domains of nearly-perfect proton-ordered 
ice monolayers on quartz may enhance deprotonation of $\mathrm{HCl}$ and $\mathrm{H}_{2} \mathrm{SO}_{4}{ }^{9}, 10,13$

Here, we follow two recent $a b$ initio studies that compared the behavior of various strong acids at the air-water interface to their bulk behavior. ${ }^{1,3}$ Experiments have stimulated such studies and have provided useful insights into the dynamical behavior of acids at aqueous surfaces. The experimental approaches have included the surface sensitive spectroscopic technique of vibrational sum frequency generation $(\mathrm{VSFG}),{ }^{14}$ and X-ray photoelectron spectroscopy (XPS). ${ }^{3}$ An alternate approach ideally suitable for examining the liquid surface structure, kinetics, and the presence of delayed as well as prompt chemical reactions is molecular scattering from a continually renewed air-liquid interface. ${ }^{15-18} \mathrm{~A}$ recent development by introducing liquid microjets has enabled the study of liquids with significant vapor pressure such as water at near-ambient temperatures. ${ }^{19,20}$ The time dependence of acid deprotonation and PT can be monitored in such studies by observing isotopic $\mathrm{D}$ to $\mathrm{H}$ exchange by mass-spectrometric detection. Inspired by this experimental development and with a view to complementing the insights to be gained in these studies, we have recently completed an ab initio study of $\mathrm{HCl}$ scattering and deprotonation at the airwater interface. ${ }^{21}$

Organic acids such as carboxylic acids RCOOH and dicarboxylic acids as well have importance in various contexts. The simplest carboxylic acids, formic acid (FA, $\mathrm{R}=\mathrm{H}$; and also acetic acid, $\mathrm{R}=\mathrm{CH}_{3}$ ) exist in significant concentrations in the environment. ${ }^{22-26}$ These carboxylic acids are some of the less reactive volatile organic compounds (VOCs) emitted by vegetation and ants for $\mathrm{FA}$, and have significant concentrations in the clean troposphere in forested areas. ${ }^{26}$ They contribute a large fraction of observed gas-phase acidity ${ }^{22,25}$ and constitute a major portion of the nonmethane organic mixture of the clean atmosphere. Furthermore, their concentrations may be increased in the polluted atmospheres in urban areas, ${ }^{25}$ where $\mathrm{FA}$ is a contributor to photochemical smog. ${ }^{23}$ Thus, they contribute to particle formation and growth, rainwater and cloud acidity, and corrosion of metals by FA. ${ }^{27}$ The acids are removed from the atmosphere through wet or dry deposition. ${ }^{23}$ Since they have a high solubility, scavenging by rainwater and wet deposition is the preferred removal route. ${ }^{25}$ Despite being weaker acids (the $\mathrm{p} K_{\mathrm{a}}$ of FA is 3.75 , the while $\mathrm{p} K_{\mathrm{a}}$ of acetic acid is 4.75) than the strong mineral acids such as sulfuric and nitric acids, the carboxylic acids usually have much larger concentrations, ${ }^{22}$ and thus may have a stronger overall effect on the free acidity of rainwater. Recently, FA has been proposed as a hydrogen storage medium for a hydrogen economy. ${ }^{28}$

Despite the prevalence of organic acids in the environment, fewer studies exist for these species, including formic acid, at interfaces. The behavior of FA in aqueous environments has been studied in both experiments and in simulations. Recent experiments have examined the protonation state of FA at the air-water interface. Brown et al. ${ }^{29,30}$ and Ottosson et al. ${ }^{31}$ have employed X-ray photoelectron spectroscopy (XPS), while the group of Johnson et al. ${ }^{32,33}$ have used VSFG. Both techniques see no or minimal deprotonation of this medium-strength acid at the air-water interface. The theoretical studies included those examining the binding energies and minimum energy structures of FA-water clusters, ${ }^{34-37}$ equilibrium and biased (metadynamics) studies of the deprotonation barrier of FA in bulk ambient water, ${ }^{38}$ classical potential studies of the free energy of solvation profile of FA at air-aqueous interfaces, including the air-water interface ${ }^{39}$ and the air-ice interface. ${ }^{40}$ The computed free energy profiles between vapor and solution ${ }^{39,40}$ support the experimentally known partitioning of FA into water, as quantified by the Henry's Law constant of $\approx 9000 \mathrm{M}$ per atm at $298 \mathrm{~K}^{41-43}$ In addition, the simulations confirm that FA has a preference for adsorption at the air-water interface, as expected by a species $\mathrm{RCOOH}$ comprised of the hydrophilic hydroxyl $(-\mathrm{OH})$ and hydrophobic R-groups.

In this work, in order to guide and assist the interpretation of ongoing experimental work, we have employed $a b$ initio simulations with density functional theory to study the mechanisms, on a timescale of a few to tens of picoseconds, of adsorption and deprotonation of formic acid at the ambient air-water interface, both with (gentle) collisions and also with biased sampling (metadynamics) to better understand the free-energy barrier to deprotonation at the interface in comparison to the bulk.

\section{Systems and methods}

\subsection{Equilibrium and collision simulations}

The QUICKSTEP module within the CP2K package ${ }^{44}$ was employed for all $a b$ initio simulations with the density functional theory (DFT) using the BLYP exchange-correlation functional ${ }^{45,46} \mathrm{com}-$ bined with a dispersion correction (BLYP-D2 ${ }^{47}$ ). The wave function was represented by double-zeta valence polarization (DZVP) basis sets on atoms in combination with GTH pseudopotentials. ${ }^{48}$ The energy cutoff for the expansion in plane waves was $280 \mathrm{Ry}$ (where 1 Ry $=0.5$ Hartree $=2.1798 \times 10^{-18} \mathrm{~J}$ ). The wave function was converged to $3 \times 10^{-7}$ Hartree. A 0.4 fs time step was used initially but then $0.5 \mathrm{fs}$ was used for the majority of the calculations. All systems were modeled with periodic boundary conditions. To reduce computational costs, the interface was modeled using a relatively thin water slab containing $72 \mathrm{H}_{2} \mathrm{O}$ molecules within a $13.4724 \times 15.5566 \times 40 \AA^{3}$ rectangular supercell, the same size as that reported in ref. 21 and $49-51$. As in previous work, ${ }^{21,52,53}$ a harmonic constraint $K\left(z-z_{0}\right)^{2}$ with a spring constant $K=1$ Hartree bohr $^{-2}\left(1 \mathrm{bohr}=0.529177 \AA, 1 \AA=10^{-10} \mathrm{~m}\right)$ was always used to ensure that the center of mass (COM) of the water slab remained at the origin of the coordinate system. Note that the constraints discussed below had this form but with a smaller spring constant, $K=0.1$ Hartree $\mathrm{bohr}^{-2}$, and were applied to the COM of FA. In addition, the system contained a single FA molecule initially placed approximately $5 \AA$ above the water surface. The amount of vacuum between adjacent images in the $z$ direction was thus 25-30 A. For comparison, calculations of FA in bulk liquid water were also performed. The model system contained a single FA and 63 water molecules in a cubic box with a side length of $12.4138 \AA$ A. All simulations were commenced using trans-FA since the cis conformer is $16.7 \mathrm{~kJ} \mathrm{~mol}^{-1}$ higher in energy (in vacuo) and not stable at ambient temperatures. ${ }^{54,55}$ The systems are displayed in Fig. 1. 
(a) Slab system

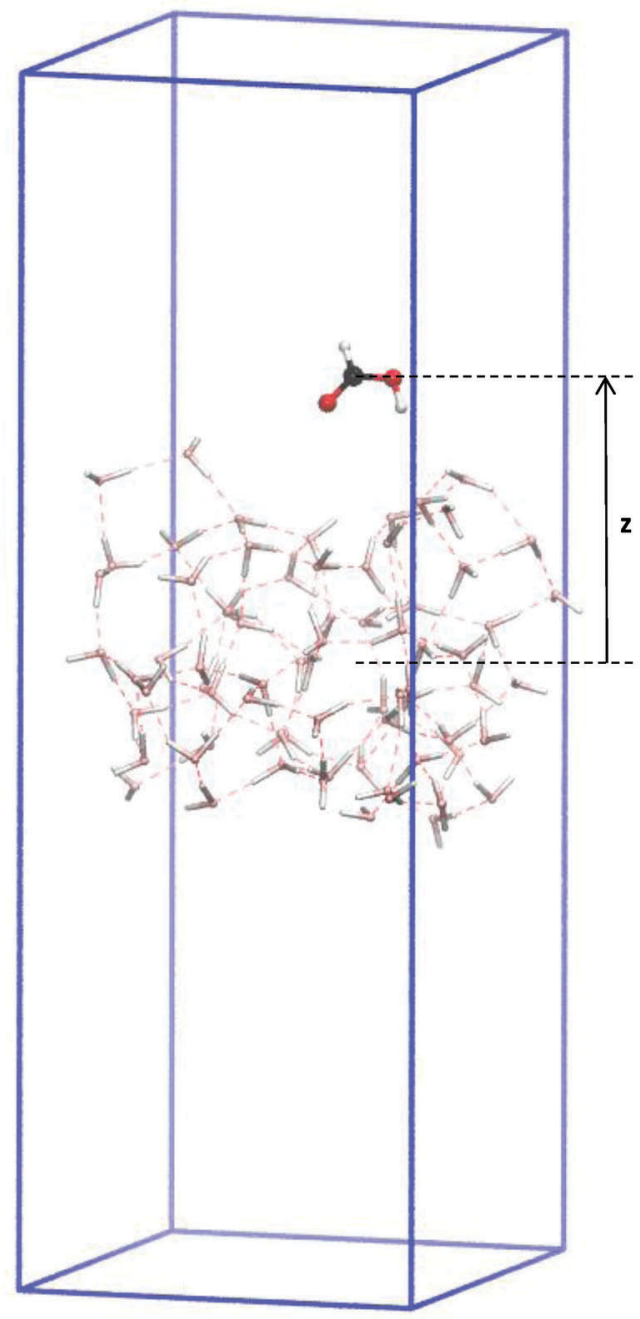

(b) FA orientation angles in the slab system

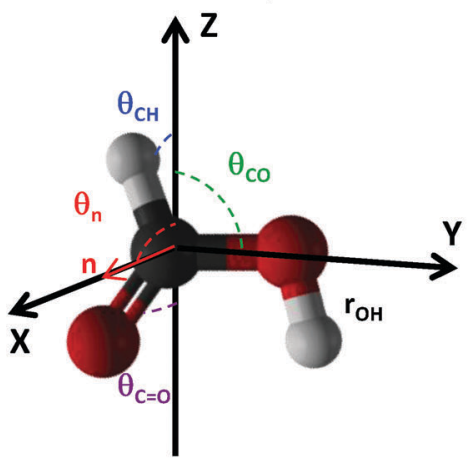

(c) Bulk system

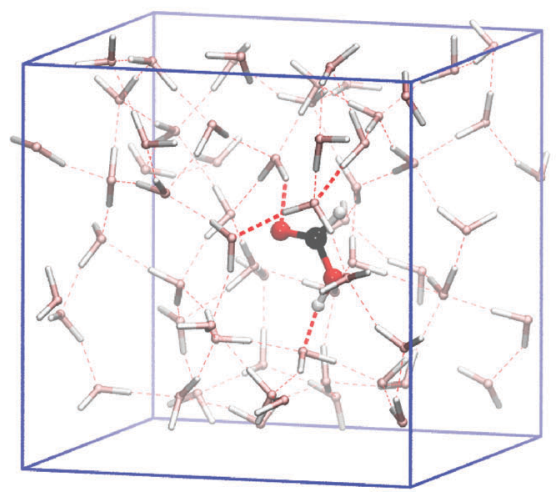

Fig. 1 Slab and bulk systems and the definition of FA orientation angles in the former system.

Prior to addition of $\mathrm{FA}$, to prepare the water systems, canonical ensemble $(N V T)$ simulations of durations $25.0 \mathrm{ps}$ and 12.0 ps using a massive Nosé-Hoover thermostat ${ }^{56,57}$ at $300 \mathrm{~K}$ were performed for the bare water slab (72 water molecules) and the bulk liquid water box ( 64 water molecules), respectively. The initial portions of the trajectories were considered to be equilibration portions, and therefore averages were computed only for the final $15.0 \mathrm{ps}$ and $10.0 \mathrm{ps}$ portions of the trajectories for the slab and the bulk liquid, respectively. For computing radial distribution functions (RDFs) and hydrogen bond (H-bond) populations for the slab, the top and bottom surfaces were averaged together. A standard geometric criterion was used to identify hydrogen bonding between two molecules with molecular groups containing a hydrogen donor and a hydrogen acceptor. ${ }^{21,52,53}$ An $\mathrm{H}$-bond was considered to exist between a donor $\mathrm{D}$ and an acceptor $\mathrm{A}$ if the distance D-A was less than $3.2 \AA$ and the $\mathrm{D}-\mathrm{H} \cdots \mathrm{A}$ angle was greater than $140^{\circ}$. With regard to the bulk water simulation, we confirmed that the internal energy per molecule or the heat of vaporization and the structure in the form of the RDFs were in good agreement with the values expected from BLYP-D simulations and with the experiment (see, e.g., ref. 53 and references therein for similar studies on water slabs and bulk water). As in ref. 52 and 53, we consider the center of the slab to be at $z=0 \AA$, and fit the density profile $\rho(z)$ to the hyperbolic tangent function

$$
\rho(z)=\frac{1}{2}\left(\rho_{1}+\rho_{\mathrm{v}}\right)-\frac{1}{2}\left(\rho_{1}-\rho_{\mathrm{v}}\right) \tanh \left(\frac{z-z_{\mathrm{GDS}}}{\delta}\right),
$$

where $\rho_{\mathrm{v}}=0 \mathrm{~g} \mathrm{~cm} \mathrm{~cm}^{-3}$ and $\rho_{\mathrm{l}}$ are the densities of the vapor and liquid phases of water. This fit yields the point where the density is half of the bulk density (the Gibbs dividing surface position $z_{\mathrm{GDS}}$ ), and the interfacial width $\delta$. We obtained $\rho_{\mathrm{l}}=1.14 \mathrm{~g} \mathrm{~cm}^{-3}, z_{\mathrm{GDS}}=4.45 \AA$, and $\delta=0.86 \AA$. As the experimental density of bulk water is $0.995 \mathrm{~g} \mathrm{~cm}^{-3}$ at $298 \mathrm{~K}$ and $1 \mathrm{~atm}$, our $10 \%$ overestimate of the liquid density reflects the relative thinness of our water slab, with only a thin bulk-like liquid central region, chosen to reduce the cost of the DFT simulations. Subsequently, we considered the bulk-like region to lie 
in $-z_{\mathrm{GDS}}+2 \delta<z<z_{\mathrm{GDS}}-2 \delta$, resulting in a $5 \AA$ thick bulk-like region. Thus, the top surface where FA will collide is $z_{\mathrm{GDS}}-2 \delta<z<z_{\mathrm{GDS}}+2 \delta$, which yields a $4 \AA$ thick interface. Fig. 2(a) shows the fitted density (only the region $z>0$ is shown). The fluctuations in the density due to capillary waves are not shown. An additional complicating factor in studies such as this one, with a soft interface, is defining the dynamic surface boundary. ${ }^{1,58}$ In this preliminary work on FA at the air-water interface, we ignore this factor in lieu of a static interface. Note that an identical water slab was used in ref. 21 to successfully model $\mathrm{HCl}$ scattering at the air-water interface.

Besides the density, the relative $\mathrm{H}$-bond population types of the water molecules at the surface are also of importance for surface reactivity. A water molecule in the bulk will prefer to form four H-bonds with its neighbors, two as a donor (D) and two as an acceptor (A). Such a water molecule is of the $\mathrm{H}$-bond type DDAA. Water molecules at the surface may prefer to be under-coordinated and may be of H-bond types ADD, DAA, DA, or A. The H-bond population profiles of the bare water slab are summarized in Fig. 2(b). These results are in good agreement with those obtained using BLYP-D2 with a thick slab. ${ }^{59}$ As expected, DDAA dominates in the center, while at the surface the largest portion of water molecules are of the type DA. Such undercoordinated water molecules account for the enhanced reactivity of the surface compared to bulk systems, assisting in molecular adsorption and reaction, as we find in our simulations. While we are using a thin slab in our definition of bulk and surface regions, it is gratifying that the main characteristics of the slab are reproduced compared to the thicker slabs used in ref. 52, 53 and 59.

Trajectories in the microcanonical ensemble $(N V E)$ of FA impacting the water slab were obtained as follows. For each trajectory, the initial geometry of the water slab came from a different snapshot (to enhance sampling) in the production portion of the NVT simulation described above. Then, the FA molecule was introduced 4-5 $\AA$ above the water surface. The COM of the FA molecule was constrained at this height and a further 2 ps $N V T$ equilibration was performed. This served to allow rotations and translations of the FA molecule that are steered by interactions with the surface to lower energy states relative to the surface since the height was close to the surface at the start for reasons of efficiency. Next, the constraint was released and the actual NVE collision simulation was performed with initial thermal velocities selected from the Maxwell-Boltzmann distribution at $300 \mathrm{~K}$. In 37 trajectories, the initial velocities of the FA molecule were purely thermal. In addition, 2 trajectories each were computed in which the COM of FA had an additional 1, 2, 4, or $10 k_{\mathrm{B}} T\left(2.5,5.0,10.0\right.$, or $24.9 \mathrm{~kJ} \mathrm{~mol}^{-1}$, respectively) of kinetic energy added, with the impact directed at $45^{\circ}$ toward the airwater interface. Thus, there were 45 trajectories computed, whose durations were mainly 8-10 ps, and 5 trajectories were extended to about 50 ps, giving about 590 ps of the total NVE trajectory time. During the trajectories, the height $z$ of the FA molecule above the surface, its acidic hydroxyl bond length $r_{\mathrm{OH}}$, and its orientation angles were monitored (see Fig. 1(b)). Other quantities that were monitored include the other bond distances, the $\mathrm{H}-\mathrm{C}-\mathrm{O}-\mathrm{H}$ dihedral angle and the possibility of trans to cis isomerization, and FA-water H-bonds.

To better understand the solvation of formic acid in various aqueous environments and also to provide starting points for the metadynamics simulations described below, we performed equilibrium simulations in the canonical ensemble $(N V T)$. Similarly to the approach used in ref. 1 and 3, we examined the activity of the interface by constraining the COM of FA at three positions in the slab, two in the interfacial region, at the surface $\left(z=z_{\mathrm{GDS}}+1 \AA\right)$ and at the subsurface $\left(z=z_{\mathrm{GDS}}-1 \AA\right)$, and at the center of the slab, at $z=0 \AA$. The last position can be compared to the analogous calculation where FA is solvated in the bulk liquid water with 63 water molecules. After equilibration, the production portions of these simulations were 14-24 ps in duration to ensure sufficient sampling.

\subsection{Metadynamics simulations}

In order to explore the deprotonation mechanisms of aqueous FA and obtain the free energy of dissociation barriers, an enhanced
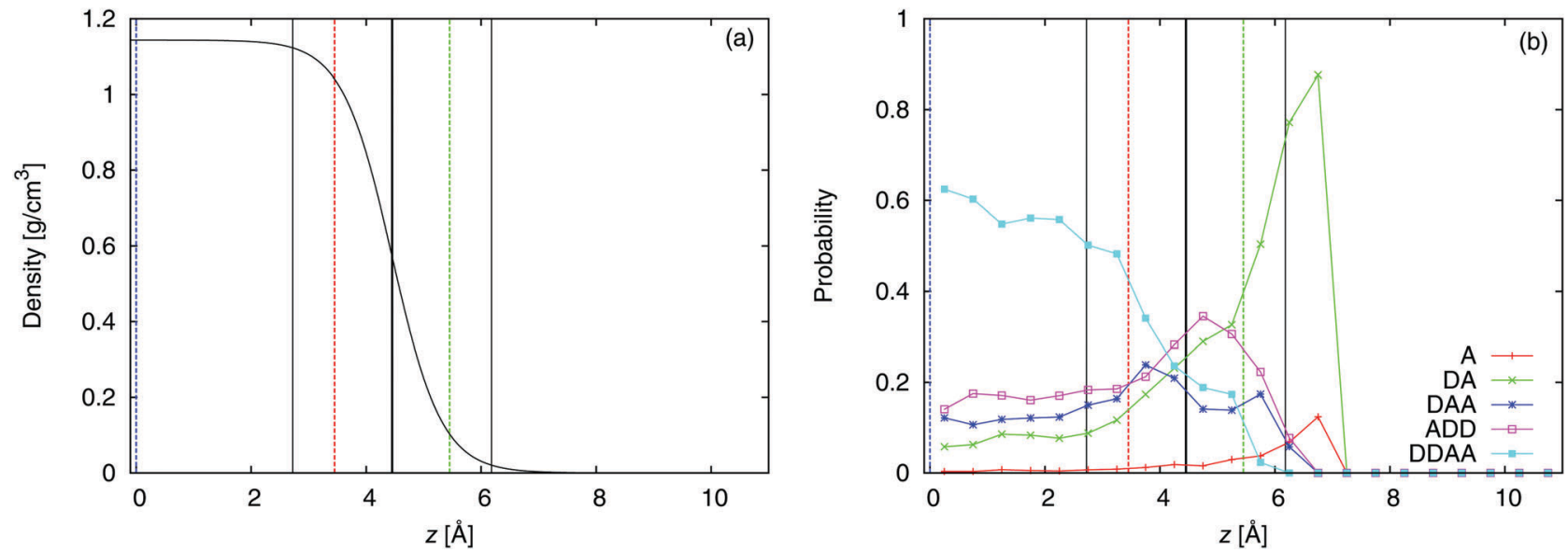

Fig. 2 (a) Density and (b) hydrogen-bonding type population profiles of water molecules in the water slab. The vertical black lines indicate the positions of $z_{\mathrm{GDS}}$ and $z_{\mathrm{GDS}} \pm 2 \delta$. The colored vertical lines refer to the metadynamics simulations with FA, marking the depths at which FA was fixed, blue, center of the slab $(z=0 \AA)$; red, subsurface region $\left(z=z_{\mathrm{GDS}}-1 \AA=3.45 \AA\right)$; and green, surface region $\left(z=z_{\mathrm{GDS}}+1 \AA=5.45 \AA\right)$. See the text for details. 
sampling method was required. We employed metadynamics, ${ }^{60,61}$ in particular, well-tempered metadynamics (WTMetaD), ${ }^{62}$ which has been shown to converge asymptotically. ${ }^{63}$ As in ref. 1 and 3, we examined the activity of the interface in various aqueous environments: bulk water, and center, subsurface, and surface of the water slab. The WTMetaD simulations always began with an equilibrated structure (with neutral, trans-FA) coming from the equilibrium simulations described above and were likewise performed in the NVT ensemble. Thus, the free energy differences are properly Helmholtz free energy differences $\Delta F$, but, with our slab setup, where the internal pressure will be approximately constant, they approximate Gibbs free energy differences $\Delta G$.

After some experimentation and tuning, the following parameters, which are typically found in ref. 62 and 63 as well, were selected. The reaction coordinate, also termed the collective variable (CV), drives the deprotonation and PT and unambiguously differentiates protonated and deprotonated states. It was chosen to be $n_{\mathrm{OH}}$, which measures the coordination of the hydroxyl oxygen of FA by its $\mathrm{H}$ atom,

$$
n_{\mathrm{OH}}=\frac{1-\left(\frac{r_{\mathrm{OH}}}{r_{0}}\right)^{n}}{1-\left(\frac{r_{\mathrm{OH}}}{r_{0}}\right)^{m}},
$$

where $r_{\mathrm{OH}}$ is the instantaneous FA hydroxyl bond length. The use of a coordination number rather than a bond length as a CV has been shown to be more robust for exploring aqueous deprotonation and PT, particularly in stabilizing and better sampling short-lived states such as the contact ion pair (CIP) and the solvent-separated ion pair (SSIP). ${ }^{64}$ The formula in eqn (2) and the parameters $r_{0}=1.6 \AA, n=6, m=12$ are chosen so that as the system undergoes transitions from neutral FA to ionized FA (CIP and SSIP), $n_{\mathrm{OH}}$ varies smoothly from about 1 (in practice about 0.9 ) to about 0 (in practice about 0.3 ). The deposition rate for Gaussian hills was 250 steps (125 fs), the Gaussian width and initial height were 0.03 and $5 \times 10^{-4}$ Hartree, respectively. For the parameter controlling the WTMetaD, we chose $\Delta T=1200 \mathrm{~K}$. The WTMetaD simulations were run for 14-18 ps. We confirmed good sampling and convergence of the relevant portions of $n_{\mathrm{OH}}$ and the relevant free energy differences, in particular the deprotonation barrier and the existence of stable CIP and SSIP states. Additional background and details of metadynamics and WTMetaD can be found in the ESI. $\dagger$

\section{Results and discussion}

\subsection{Collision simulations}

We will discuss mainly six representative thermal collision trajectories: two trajectories where spontaneous dissociation was observed (labeled 1 and 2) and four trajectories where it was not (labeled 3-6). Trajectory 1 was 10 ps in duration, while trajectories 2-5 lasted 50 ps. The collisions with added kinetic energy had similar outcomes, with the exception that they took longer to equilibrate to the surface, and thus had a reduced likelihood for deprotonation within the duration of the computed

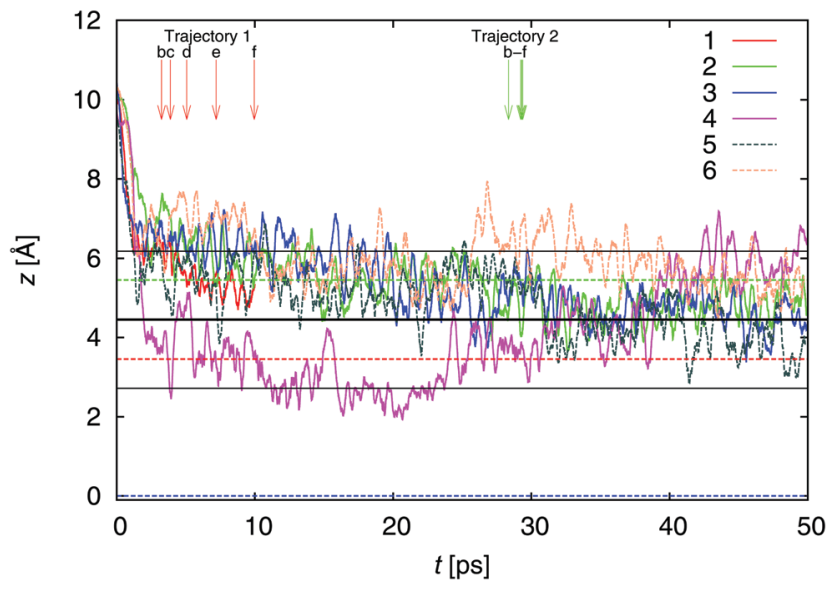

Fig. 3 The vertical position of FA carbon atom relative to the center of the water slab is shown for collision trajectories $1-6$. The black lines mark the position of the Gibbs dividing surface $z_{\mathrm{GDS}}$ of the water slab and the interfacial width $\pm 2 \delta$ of the surface. The colored dotted lines (blue, center of the slab; red, subsurface region; and green, surface region, see Fig. 2) mark the vertical positions at which FA was fixed in the metadynamics simulations and are drawn here to show how often the unrestrained trajectories visit these regions. The arrows labeled $b-f$ refer to the snapshots shown in Fig. 5 (trajectory 1) and 6 (trajectory 2).

trajectory. In our study of $\mathrm{HCl}$ at the air-water interface, ${ }^{21}$ it was observed that adding energy to the system, either by raising the temperature of the water slab, or by adding collision energy to $\mathrm{HCl}$, typically reduced the time required for $\mathrm{HCl}$ to deprotonate. With formic acid, this seems not to be the case either because FA is a weak acid and more collision energy is needed or because the energy can be distributed among more modes of the larger molecule. The results for all 45 scattering trajectories can be found in the ESI. $\dagger$

3.1.1 Formic acid height above the water surface, its hydroxyl bond length, and sample trajectory snapshots. Fig. 3 shows the approach of FA to the water slab. With our definition of the interfacial region being $z_{\mathrm{GDS}}-2 \delta<z<z_{\mathrm{GDS}}+2 \delta$, all trajectories resulted in FA adsorption at the interface within about 2 ps. FA remained in the interfacial region even in the longest duration trajectories of 50 ps. No trajectories resulted in FA scattering, absorption into the bulk or desorption within up to $50 \mathrm{ps}$. Adsorption is to be expected since $50 \mathrm{ps}$ is likely too short a duration to observe diffusion into the bulk leading to absorption and certainly much too short to see desorption. In our study ${ }^{65}$ modeling the pickup of a non-hydrogen-bonding molecule, $\mathrm{NO}_{2}$, at the water surface, using classical potentials, we did not observe much absorption or desorption until the duration of the simulations reached about 100 ps. Moreover, nearly 200 trajectories were computed. The required simulation durations/statistics to observe these processes for FA at the water surface would be much longer given that much stronger bonding, FA-water $\mathrm{H}$-bonds, is present in this system, and such an effort using $a b$ initio potentials is outside the scope of this study. However, regardless of the simulation duration, an adsorption preference of FA on water is nevertheless expected given the known surface activity of FA seen both in other simulations and experimentally. 
Pártay et $a{ }^{39}{ }^{39}$ in a classical potential study calculated the free energy of solvation profile of FA at the air-water interface and obtained free energies for adsorption and absorption of $-24.7 \mathrm{~kJ} \mathrm{~mol}^{-1}$ and $-16.7 \mathrm{~kJ} \mathrm{~mol}^{-1}$, respectively, relative to FA in the gas phase. Note that this study used rigid monomer force fields and the results would likely change somewhat if ab initio potentials were used. However, with this caveat, the results reported by Pártay et al. imply that at equilibrium and at infinite dilution the partitioning of FA is about $2 \times 10^{4}: 1$ (adsorbed : vapor), $1 \times 10^{3}: 1$ (absorbed:vapor), and 20:1 (adsorbed:absorbed). Our results are in general agreement with these partitioning predictions. Experimentally, the Henry's Law constant ${ }^{43}$ of $9000 \mathrm{M}$ per atm at $25{ }^{\circ} \mathrm{C}$ also reflects the high solubility of FA, although it does not distinguish between adsorption in the surface region and absorption into the bulk. We note that the trapping observed in all collisions and the absence of desorption over the 50 ps trajectories is also in accord with separate molecular beam scattering experiments ${ }^{19,20}$ of FA collisions with 8 molal $\mathrm{LiBr} /$ $\mathrm{H}_{2} \mathrm{O}$ at $250 \mathrm{~K}$. These microjet experiments show that almost every FA impinging at thermal collision energies disappears into solution, and does not evaporate over the 100 microsecond observation window. ${ }^{66}$

Fig. 4 presents the formic acid hydroxyl bond length $r_{\mathrm{OH}}$ as a function of time for the six selected trajectories. The dotted line and labels on the right axis mark the approximate stretched $\mathrm{O}-\mathrm{H}$ bond lengths when an $\mathrm{H}$-bond is formed through donation of the $\mathrm{H}$ to a water oxygen atom $\left(r_{\mathrm{OH}}=1.1 \AA\right)$, when the proton is shared $(1.4 \AA)$, when dissociation occurs and a contact ion pair or CIP is formed (1.6 $\AA$ ), and when a minimal-distance solventseparated ion pair or SSIP is formed $(1.8 \AA)$. Fig. 4 shows that while a number of trajectories exhibited stretched FA (O)H bonds, only two of the 45 collision trajectories (4\%) underwent spontaneous deprotonation.

Fig. 5 shows snapshots from trajectory 1 (top view). FA first establishes an (O)H donor hydrogen bond (H-bond, panel (a)) with a surface-water oxygen atom. FA then deprotonates at $3.88 \mathrm{ps}$. At this time, FA is solvated by three H-bonds, the mentioned

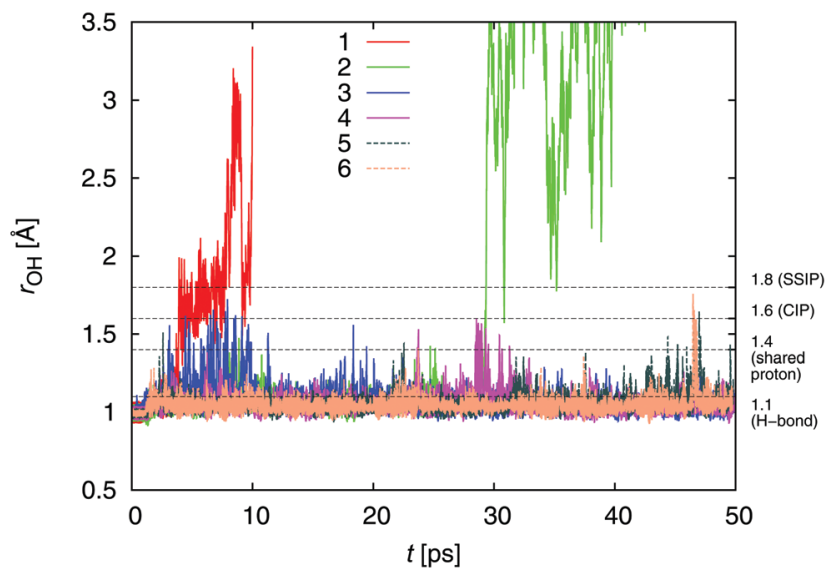

Fig. 4 The $\mathrm{O}-\mathrm{H}$ separation in FA is shown for collision trajectories 1-6. The dashed black lines mark the approximate $\mathrm{O}-\mathrm{H}$ separations for various states of aqueous FA. See the text for details. donor H-bond of FA and two H-bonds accepted by FA from nearby water molecules at $(\mathrm{C}=)$ O. Panels $(\mathrm{b}-\mathrm{f})$ show the deprotonation and formation of the CIP and SSIP as PT proceeds. Both Eigen $\left(\mathrm{H}_{3} \mathrm{O}^{+}\right)$and Zundel $\left(\mathrm{H}_{5} \mathrm{O}_{2}{ }^{+}\right)$charged water species were present. To summarize, following deprotonation, the proton defect migrates through a ring of four water molecules and neutral FA is reformed in the cis conformation. The whole process is completed within approximately 6 ps. In Fig. 3, the red arrows for trajectory 1, marked b-f, indicate that the entire deprotonation and reprotonation process occurs near the top of the interface, $z \sim z_{\mathrm{GDS}}+1$, mainly $z>z_{\mathrm{GDS}}$, what we refer to as the "surface" region.

Fig. 6 shows snapshots from trajectory 2. Note the differences in FA orientations exhibited between the panels showing the start of the trajectory (a) and the H-bond prior to dissociation at (b). Likely due to the necessity to establish an optimum orientation and solvation, this trajectory required a long wait before the deprotonation process was observed at $29.230 \mathrm{ps}$ but PT was rapid once it commenced. Panel (c) shows that at dissociation FA exhibits a tilt relative to the surface normal. As in trajectory 1, FA is involved in three $\mathrm{H}$-bonds with surface water molecules. However, here it donates $(\mathrm{O}) \mathrm{H}$ and accepts one $\mathrm{H}$-bond at $\mathrm{O}(\mathrm{H})$ and one $\mathrm{H}$-bond at $(\mathrm{C}=)$ O. Following deprotonation, the proton defect migrates through a wire of three water molecules. Neutral FA is reformed in the trans conformation but with the proton attached to the other FA oxygen atom. The whole ultrafast process is completed in about 120 fs. In Fig. 3, the green arrows for trajectory 2, marked b-f, indicate that again this deprotonation-reprotonation process occurs within the top of the interface.

3.1.2 Formic acid deprotonation and proton transfer dynamics. Collision trajectories 1 and 2 exhibited deprotonation with quite dissimilar timescales. Unlike in trajectory 1 and also the other deprotonation processes observed in equilibrium and metadynamics simulations (see below), the extremely fast nature of the process in trajectory 2 indicates that it is an example of concerted PT, i.e., the simultaneous, cooperative motion of two or more protons. The mechanisms and dynamics of concerted PT in aqueous systems, ${ }^{67-72}$ particularly in icy ${ }^{73-75}$ and also in biological systems, ${ }^{76}$ are of interest both from theoretical and experimental standpoints. ${ }^{67-75,77}$ While concerted PT has been known to be present in icy systems, the Grotthuss mechanism ${ }^{77-79}$ in liquid water has long been thought to be dominated by sequential PT events. However, recently the Grotthuss mechanism in liquid water has been reinvestigated, and concerted PT is also believed to occur in liquid water, where proton migration is now thought to encompass both sequential PT events, separated by rest periods, and concerted PT of two or more protons through a closely-spaced water wire. ${ }^{67}$

To better understand how the issues discussed impact our system, we performed a detailed structure and dynamics analysis of the deprotonation and PT events in trajectories 1 and 2. This analysis is shown graphically in Fig. 7 and 8 .

In trajectory 1 , as mentioned above, Fig. 7 shows that the deprotonation of trans-FA, the series of PT events, and reprotonation processes ending in neutral cis-FA occur via PT through a ring of four $\mathrm{H}$-bonded water molecules attached to the hydroxyl 
(a) $t=0.00$ ps: Released above surface

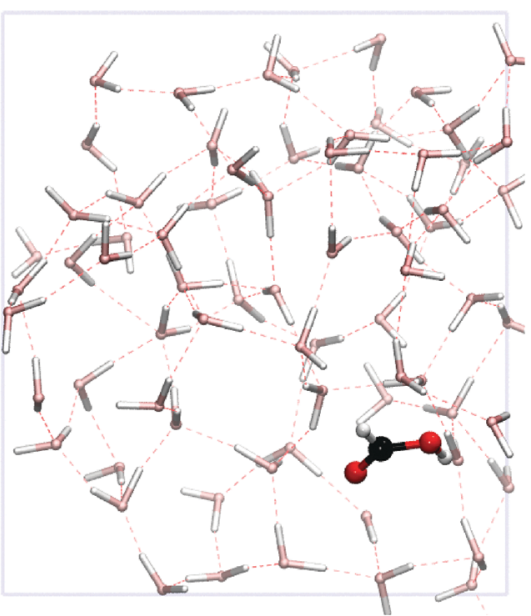

(d) $t=5.07$ ps: Solvent-Separated

Ion Pair (SSIP) (with Eigen cation)

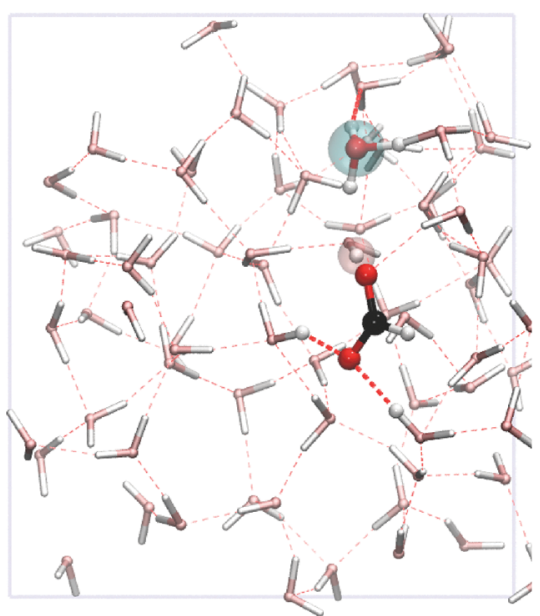

(b) $t=3.24$ ps: H-bond

before dissociation

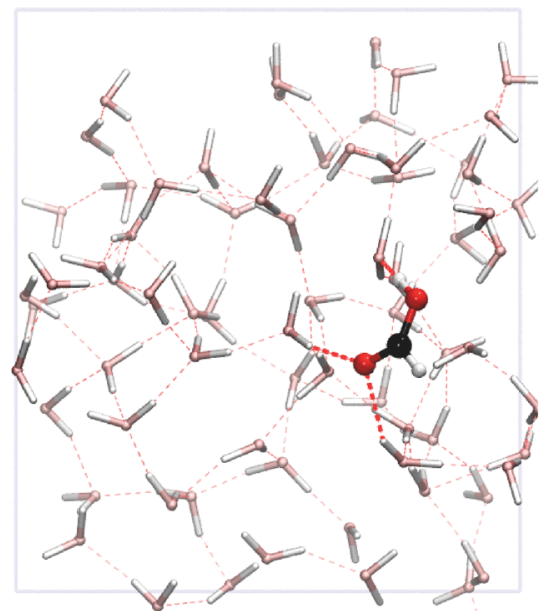

(e) $t=7.20$ ps: CIP2

(Shared proton)

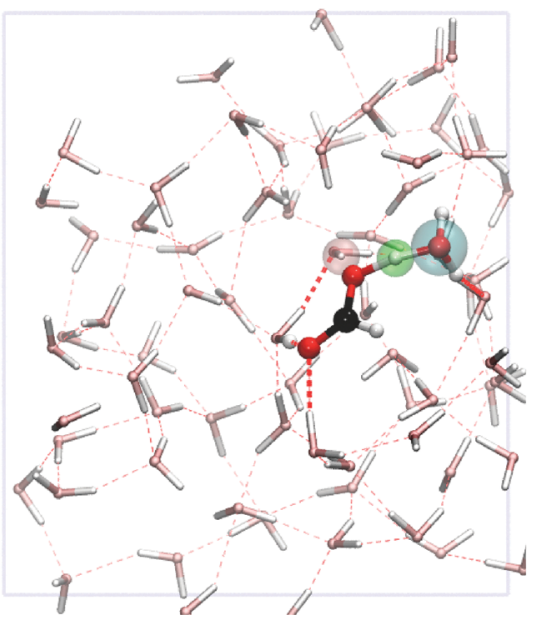

(c) $t=3.88$ ps: Contact Ion

Pair (CIP) (with Eigen cation)

Fig. 5 Snapshots (top view) from trajectory 1 showing FA deprotonation and PT at the air-water interface. Key used throughout this paper: carbon, black; oxygen, red; hydrogen, white; background atoms, pastel colors; hydrogen bonds shown with dotted red lines. Important atoms or states are highlighted by bubbles: (1) cyan bubble (large) - indicates an Eigen (one cyan bubble) or Zundel (two cyan bubbles) species produced after FA deprotonation and proton migration; (2) red bubble - initial proton from FA after FA deprotonation; (3) green bubble - another proton which covalently attaches to the same FA oxygen atom (making neutral FA again, either trans or cis); (4) blue bubble (not in this figure but present in, e.g., trajectory 2, see Fig. 6) - another proton which covalently attaches to the other FA oxygen atom (making neutral FA again, either trans or cis).

oxygen, $\mathrm{O}(\mathrm{H})$ (here labeled $\mathrm{O}(\mathrm{I})$; similarly the carbonyl oxygen $(\mathrm{C}=) \mathrm{O}$ is labeled $\mathrm{O}(\mathrm{II})$ ); see panel (a) which displays a snapshot at $t=4.665 \mathrm{ps}$. While in bulk ice and at lower temperatures PT may rely mainly on proton tunneling, water molecule mobility due to thermal fluctuations in liquid water (and even the greater mobility of water molecules at the surface of defect-rich ice ${ }^{11}$ or at the ice quasi-liquid layer ${ }^{12}$ ) permits the occasional shrinkage of $\mathrm{O}-\mathrm{O}$ distances which significantly reduces the barrier to PT, and allows the process to proceed without invoking proton tunneling. ${ }^{77,79}$ The sequences of $\mathrm{O}-\mathrm{O}$ distances and PT coordinates in Fig. $7(\mathrm{a}-\mathrm{c})$ are defined as $r_{\mathrm{OO}, i}=\left|\mathbf{r}_{\mathrm{O}, i-1}-\mathbf{r}_{\mathrm{O}, i}\right|$ and $\xi_{i}=\left|\mathbf{r}_{\mathrm{O}, i-1}-\mathbf{r}_{\mathrm{H}, i}\right|-\left|\mathbf{r}_{\mathrm{H}, i}-\mathbf{r}_{\mathrm{O}, i}\right|$. Thus, for a proton $i, \xi_{i}<0, \xi_{i}=0$, and $\xi_{i}>0$ prior to, at the moment of, and after the PT event, respectively.
The insets in panels (b) and (c) of Fig. 7 focus attention on the PT events. It it seen that the $i$ th proton is transferred when $r_{\mathrm{OO}, i} \approx 2.4 \AA$. The PT of protons 1 and 2 is nearly concerted. The remaining PT events are sequential, separated by rest periods. This is in agreement with the new understanding of PT events in liquid water: ${ }^{67}$ containing both intense bursts of activity in the form of concerted transfers, on a timescale of tens to hundreds of femtoseconds, and sequential PT events as well, separated by rest periods, resulting in a slower, few picosecond timescale. Here, the timescale of the overall process is dominated by sequential transfers/rest periods, and thus occupies approximately 6 ps. Since the ring of water molecules starts and ends on $\mathrm{O}(\mathrm{I})$, the overall result of these PT events is to transform trans-FA into cis-FA. 
(a) $t=0.000$ ps: Released above

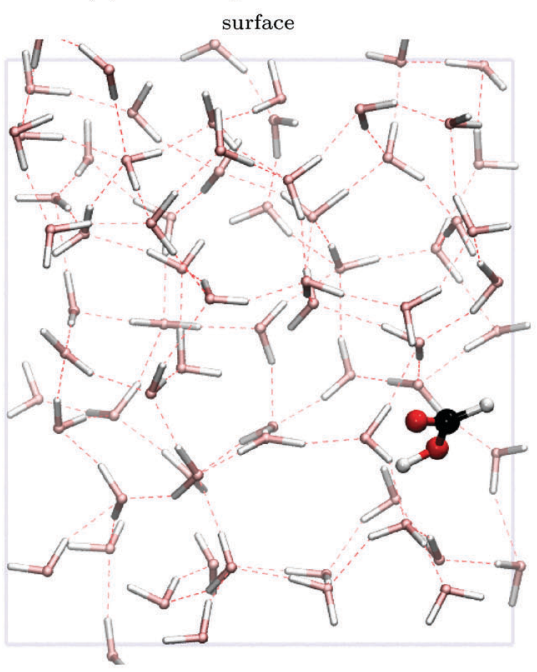

(d) $t=29.307$ ps: CIP2 (with Zundel cation)

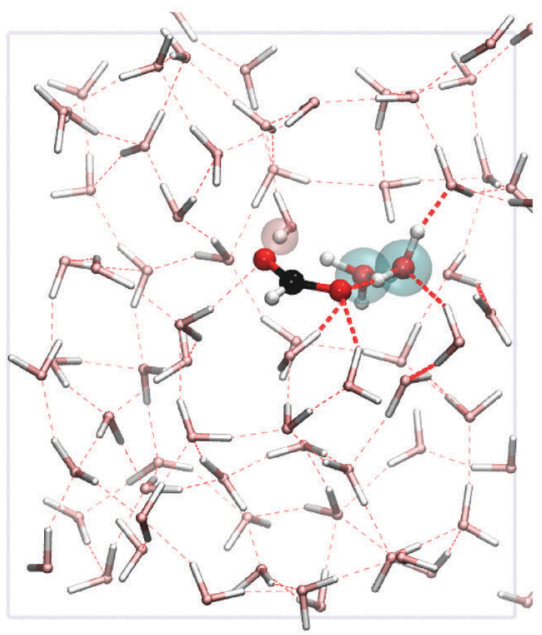

(b) $t=28.348$ ps: H-bond before dissociation

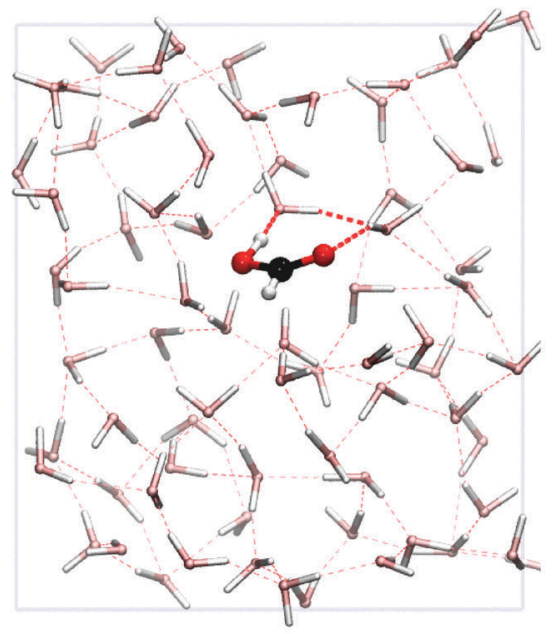

(e) $t=29.308$ ps: CIP2 (with Eigen cation)

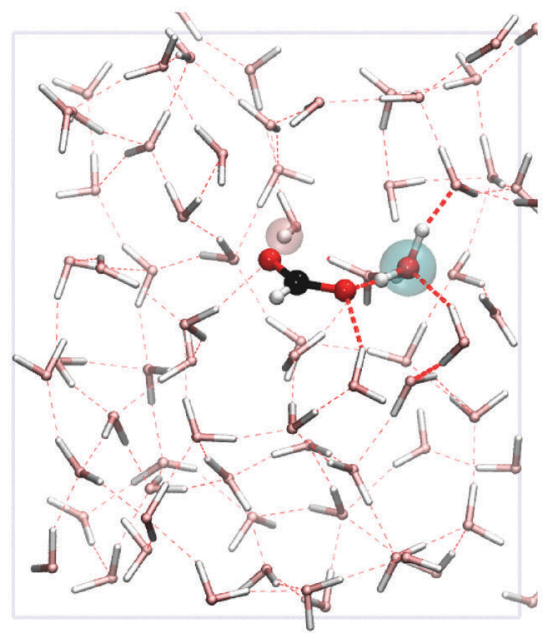

(c) $t=29.230$ ps: Contact Ion

Pair 1 (CIP) (with Eigen cation)

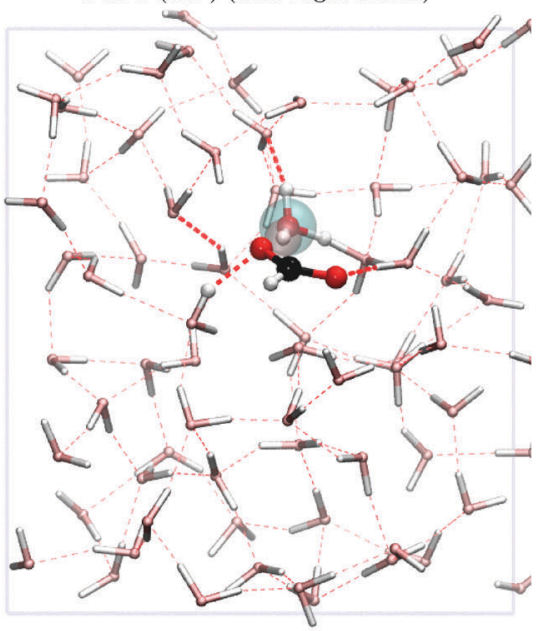

(f) $t=29.377$ ps: Neutral (trans) reformed with $\mathrm{H}^{+}$on other $\mathrm{O}$

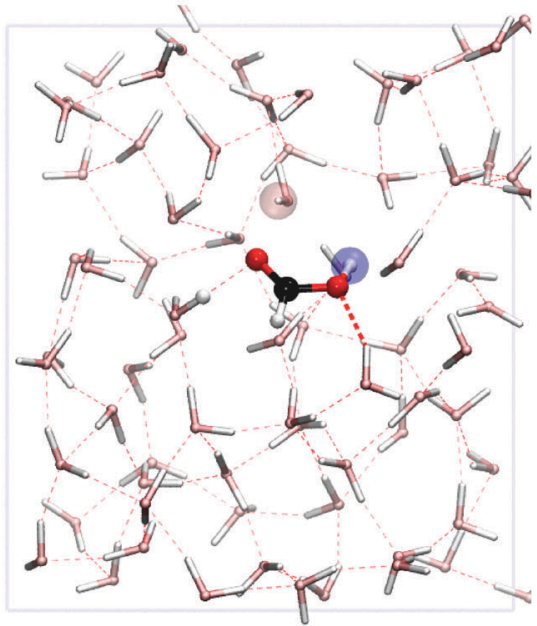

Fig. 6 Snapshots (top view) from trajectory 2 showing the FA deprotonation and PT at the air-water interface. Also see Fig. 5.

In trajectory 2, Fig. 8 shows that the deprotonation of trans-FA, the series of PT events, and reprotonation processes ending again in neutral trans-FA occur via PT through a wire of three H-bonded water molecules connecting $\mathrm{O}(\mathrm{I})$ to $\mathrm{O}(\mathrm{II})$; see panel (a) which displays a snapshot at $t=29.293$ ps. Such an arrangement is known to be a low-energy configuration from gas phase (cluster) experimental and theoretical studies, with particularly strong and therefore short H-bonds. ${ }^{35}$ The sequences of O-O distances $r_{\mathrm{OO}, i}$ and PT coordinates $\xi_{i}$ in Fig. 8(a-c) are defined similarly as in Fig. 7(a-c).

The long wait for the PT events in trajectory 2 can be partially understood by seeing that in panel (b) of Fig. 8 the relative distance between water molecules 2 and 3 is initially large. Thus, the wire of three waters facilitating PT in this trajectory takes a long time to assemble. Once the wire is assembled, panel (c) shows that all the PT events are ultrafast: there is concerted transfer of protons 2 and 3 and nearly concerted transfer of the other protons. Thus, concerted PT is fully dominant in this trajectory and only the shorter timescale is relevant, allowing the whole process to be completed in about 120 fs. The result of the overall PT events is to reform trans-FA, while switching the identity of the hydroxyl and carbonyl oxygen atoms.

3.1.3 Formic acid $\mathbf{H}$-bonds with water. Fig. 9 shows the $\mathrm{H}$-bonds that formic acid forms with nearby water molecules for trajectories 1-4. As FA approaches the water surface, the number of $\mathrm{H}$-bonds typically rises, with the initial $\mathrm{H}$-bond most often being the hydroxyl donor bond to water, (O) $\mathrm{H} \cdots \mathrm{Ow}$. Additional H-bonds can be formed as FA equilibrates to the surface and establishes a solvation shell, including an acceptor $\mathrm{H}$-bond at the carbonyl oxygen, $(\mathrm{C}=) \mathrm{O} \cdots \mathrm{Hw}$. If FA sinks deeper into the water surface and the solvation shell continues to form, FA can accept up to two additional relatively weak $\mathrm{H}$-bonds at each oxygen atom. These observations are in agreement with experimental and theoretical studies of FA microsolvation in small water clusters, where it is seen that one strong and typically 1-2 or 3 weaker $\mathrm{H}$-bonds with water molecules can be formed. ${ }^{34,35}$ The rightmost panels in Fig. 9 show the total number of $\mathrm{H}$-bonds that FA establishes with water. It is seen 

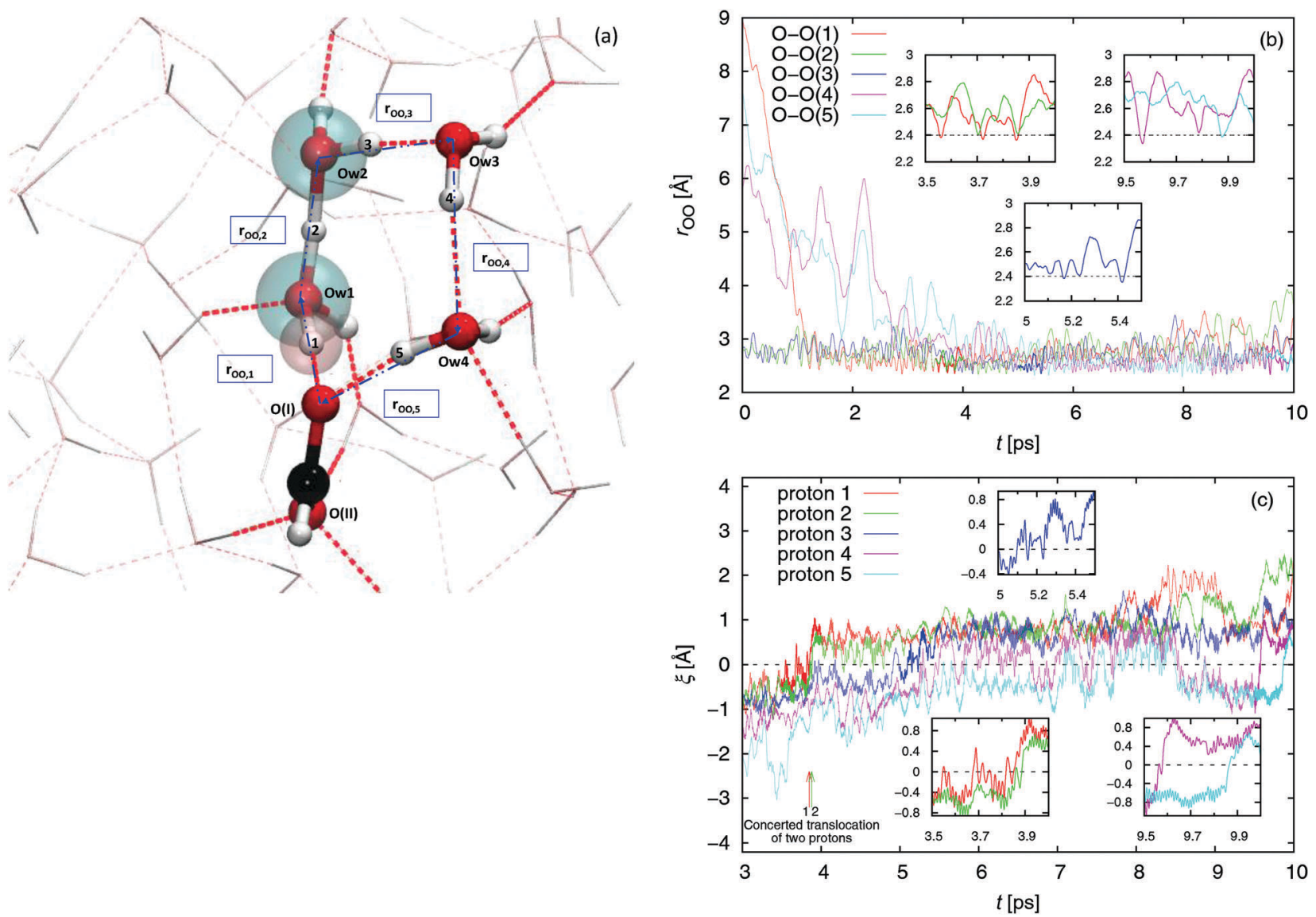

Fig. 7 Proton transfer dynamics in trajectory 1. Panel (a) shows a snapshot at $t=4.665$ ps when deprotonation has occurred and formate is solvated by five $\mathrm{H}$-bonds and the proton is delocalized ("rattling") over two water molecules (Zundel cation, $\mathrm{H}_{5} \mathrm{O}_{2}{ }^{+}$). The blue arrows indicate the direction of PT and the O-O distances $r_{\mathrm{OO}, \mathrm{i}}$. The five consecutive protons involved in PT are numbered. Proton 1 comes from FA, while protons $2-5$ are from the attached ring of four water molecules. The $\approx 6$ ps process begins with trans-FA $(t \approx 3.9 \mathrm{ps})$ and terminates with cis-FA $(t \approx 9.9 \mathrm{ps})$. Panel (b) shows $r_{\mathrm{OO}, i}$ with time for the whole trajectory, with the insets emphasizing the PT events. The dotted lines at $2.4 \AA$ in the insets mark the approximate distance for the lowering of the PT barrier which triggers deprotonation. Panel (c) shows the PT coordinates $\xi_{i}$ with time for the important portion of the trajectory, with the insets emphasizing the PT events. It is seen that protons 1 and 2 are transferred simultaneously, while the other transfers occur sequentially and are separated in time by rest periods.

that the two instances of deprotonation, in trajectories 1 and 2, are often correlated with FA forming about $4 \mathrm{H}$-bonds. However, such an H-bonding or solvation state does not always lead to deprotonation. Deprotonation and Grotthuss migration of the proton defect also depends on the state of the second solvation shell surrounding the acid. ${ }^{78}$ This is in agreement with the behavior of other acids whose deprotonation also depends on some minimal solvation. ${ }^{80}$

\subsection{Equilibrium simulations}

To better understand the state of formic acid in the various aqueous environments, we also performed equilibrium simulations with formic acid in bulk liquid water and constrained at three depths (center and subsurface and surface of the slab). The results of the simulations are described below. We observed spontaneous dissociation when formic acid was at the subsurface (see the $\mathrm{ESI} \dagger$ for details).

3.2.1 Formic acid forms $\mathbf{H}$-bonds with water at equilibrium. Table 1 shows the average number of $\mathrm{H}$-bonds that FA forms with water molecules. In bulk liquid water (63 water molecules),
FA forms approximately $3.3 \mathrm{H}$-bonds, while at the center of the slab we obtain $2.8 \mathrm{H}$-bonds. These numbers are in reasonable agreement given the large fluctuations/uncertainty shown in the table, which is partially due to the (discontinuous) definition of an H-bond used in this work. This is in good agreement with the number of $\mathrm{H}$-bonds $(\approx 3)$ and consistent with the water hydration numbers (4.6-4.9) obtained in other simulations ${ }^{81,82}$ and extracted from experiments. ${ }^{83-85}$

At the surface and subsurface of the slab, the number of H-bonds was slightly reduced, to 3.0. After the spontaneous deprotonation at the subsurface, however, the average number of H-bonds that formate participated in was 4.1, in reasonable agreement with ref. 81 and other studies. ${ }^{82-85}$ Such an increase in H-bonds and hydration numbers of the anion compared to the neutral acid is expected since the anion can form stronger $\mathrm{H}$-bonds, and thus better compete with water-water H-bonds.

3.2.2 Formic acid orientation at the air-water interface. The histograms in Fig. 10 obtained from equilibrium simulations permit more precise statements about the orientational preferences of neutral trans-FA in the interfacial region. Two orientational 

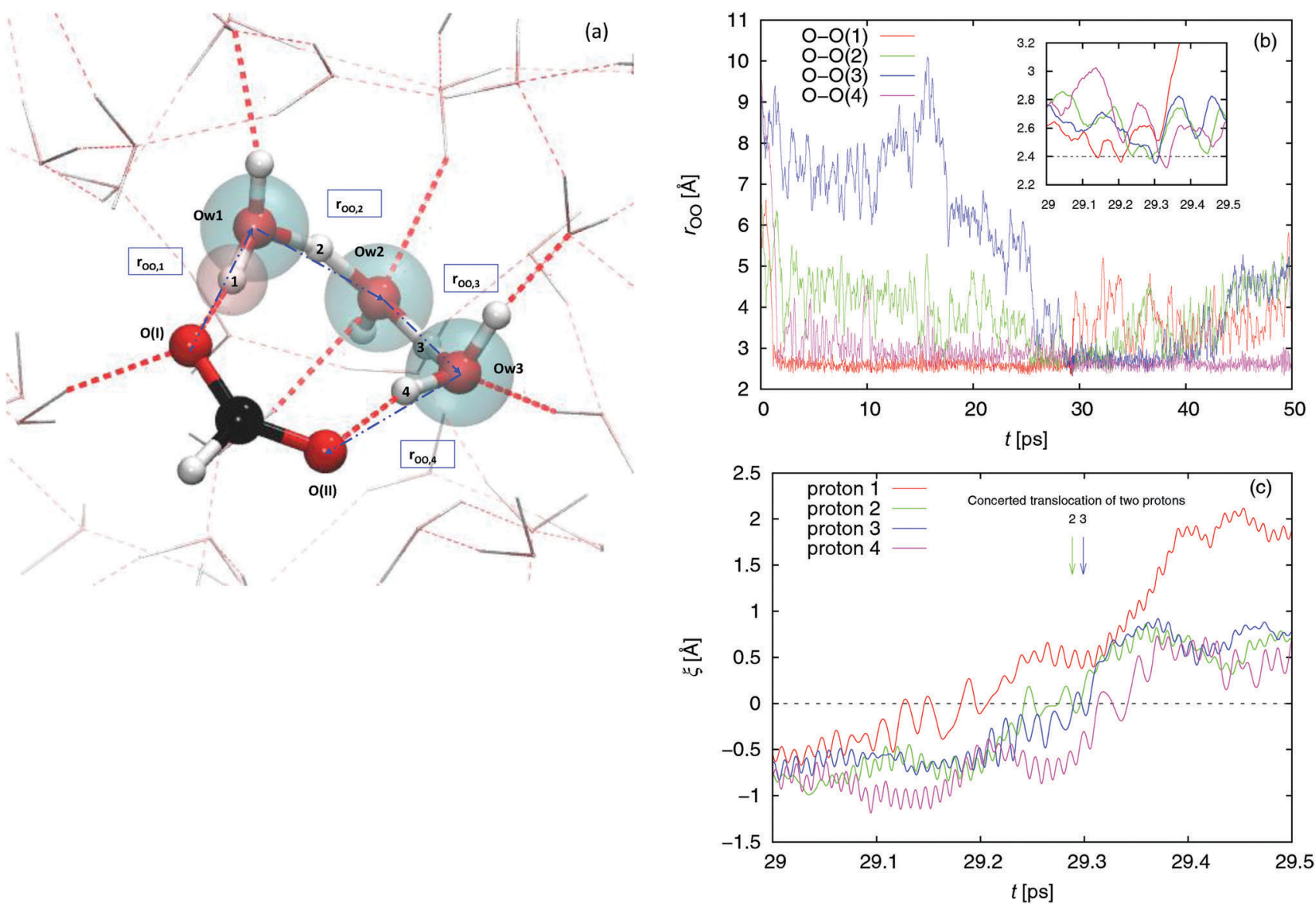

Fig. 8 Proton transfer dynamics in trajectory 2. Panel (a) shows a snapshot at $t=29.293 \mathrm{ps}$ when deprotonation has occurred and formate is solvated by three $\mathrm{H}$-bonds and the proton defect is delocalized ("rattling") over three water molecules $\left(\mathrm{H}_{7} \mathrm{O}_{3}{ }^{+}\right)$. The blue arrows indicate the direction of PT and the $\mathrm{O}-\mathrm{O}$ distances $r_{\mathrm{OO}, i}$. The four consecutive protons involved in PT are numbered. Proton 1 comes from FA, while protons 2-4 are from the attached wire of three water molecules. The $\approx 120 \mathrm{fs}$ process begins with trans-FA ( $t \approx 29.230 \mathrm{ps}$ ) and terminates with cis-FA ( $t \approx 29.350 \mathrm{ps})$. Panel (b) shows $r_{\mathrm{OO}, i}$ with time for the whole trajectory, with the inset emphasizing the PT events. The dotted line at $2.4 \AA$ in the inset marks the approximate distance for the lowering of the PT barrier which triggers deprotonation. Panel (c) shows the PT coordinates $\xi_{i}$ with time for the important portion of the trajectory. It is seen that protons 2 and 3 are transferred simultaneously, while the other transfers occur sequentially and are separated in time by short rest periods.

angles are presented, as defined in Fig. 1 (see the ESI $\dagger$ for more details). At the surface, the orientation is primarily vertical. This is in agreement with the results reported by Johnson et $a l .^{32}$ who used VSFG spectroscopy of FA at the air-water interface to determine that the orientation angles of undissociated trans-FA were $\theta_{\mathrm{CH}}^{\exp }=27-43^{\circ}$ and $\theta_{\mathrm{C}}^{\exp }{ }_{\mathrm{O}}=42-57^{\circ}$. Since VSFG is most sensitive to molecules at the top of interfaces, this can be compared to our values of $\theta_{\mathrm{CH}}^{\text {surface }}=42 \pm 12^{\circ}$ and $\theta_{\mathrm{C}=\mathrm{O}}^{\text {surface }}=38 \pm 14^{\circ}$. In the subsurface region, FA undergoes a rotation to a tilted orientation, with $\theta_{\mathrm{CH}}^{\text {subsurface }}=83 \pm 18^{\circ}$ and $\theta_{\mathrm{C}}^{\text {subsurface }}=78 \pm 16^{\circ}$. Such a rotation may be energetically favored since it will place the carbonyl $(-\mathrm{C}=\mathrm{O})$ group of FA closer to the under-coordinated water molecules present at the surface, thereby allowing for $(\mathrm{C}=) \mathrm{O} \cdots \mathrm{Hw} \mathrm{H}$-bond formation without necessitating a competition with other water molecules. Comparing the relatively sharp orientational angle distributions of FA at the surface to the broader ones at the subsurface, it is seen that an average increase in depth of merely $2 \AA$ quickly causes loss of a well-defined orientational preference. Thus, it may be that the VSFG spectroscopic method used by Johnson, dependent as it is on a well-defined molecular orientation and insensitive to a homogeneous environment, does not detect the subsurface FA where deprotonation is most enhanced (see below) due to this rapid loss of orientational preference.

\subsection{Metadynamics simulations}

Fig. 11 presents the results of the WTMetaD simulations of FA deprotonation. We sampled sufficiently to explore the configurational space and obtain convergence (see ESI $\dagger$ ). Fig. 11 shows the free energies and barriers for the deprotonation and PT processes in the configurational snapshots. The states of the system can be characterized by the value of $\mathrm{CV} n_{\mathrm{OH}}$ defined in eqn (2) (or, equivalently, $r_{\mathrm{OH}}$, which is shown on the top axis). These system states are: state a - the FA initial neutral or the FA (O)H hydrogen bonded to a water oxygen atom, $n_{\mathrm{OH}} \approx 0.9$ $\left(r_{\mathrm{OH}} \approx 1.1 \AA\right.$ ) ; state $\mathrm{b}$ - the shared-proton configuration, $n_{\mathrm{OH}} \approx 0.7$ $\left(r_{\mathrm{OH}} \approx 1.4 \AA\right)$; state $\mathrm{c}$ - the contact-ion pair $(\mathrm{CIP}), n_{\mathrm{OH}} \approx 0.5\left(r_{\mathrm{OH}} \approx\right.$ $1.6 \AA)$; and state $d$ - the closest solvent-separated ion pair (SSIP), $n_{\mathrm{OH}} \approx 0.3\left(r_{\mathrm{OH}} \approx 1.8 \AA\right.$ A). The largest barrier to deprotonation (the transition state marked $\mathrm{TS}_{\mathrm{ab}}$ ) is located between states $\mathrm{a}$ and $\mathrm{b}$. 
FA hydroxyl donates and accepts: $(\mathrm{O}) \mathrm{H}-\mathrm{Ow}$ and $\mathrm{O}(\mathrm{H})-\mathrm{Hw}$
FA carbonyl accepts: $(\mathrm{C}=) \mathrm{O}-\mathrm{Hw}$

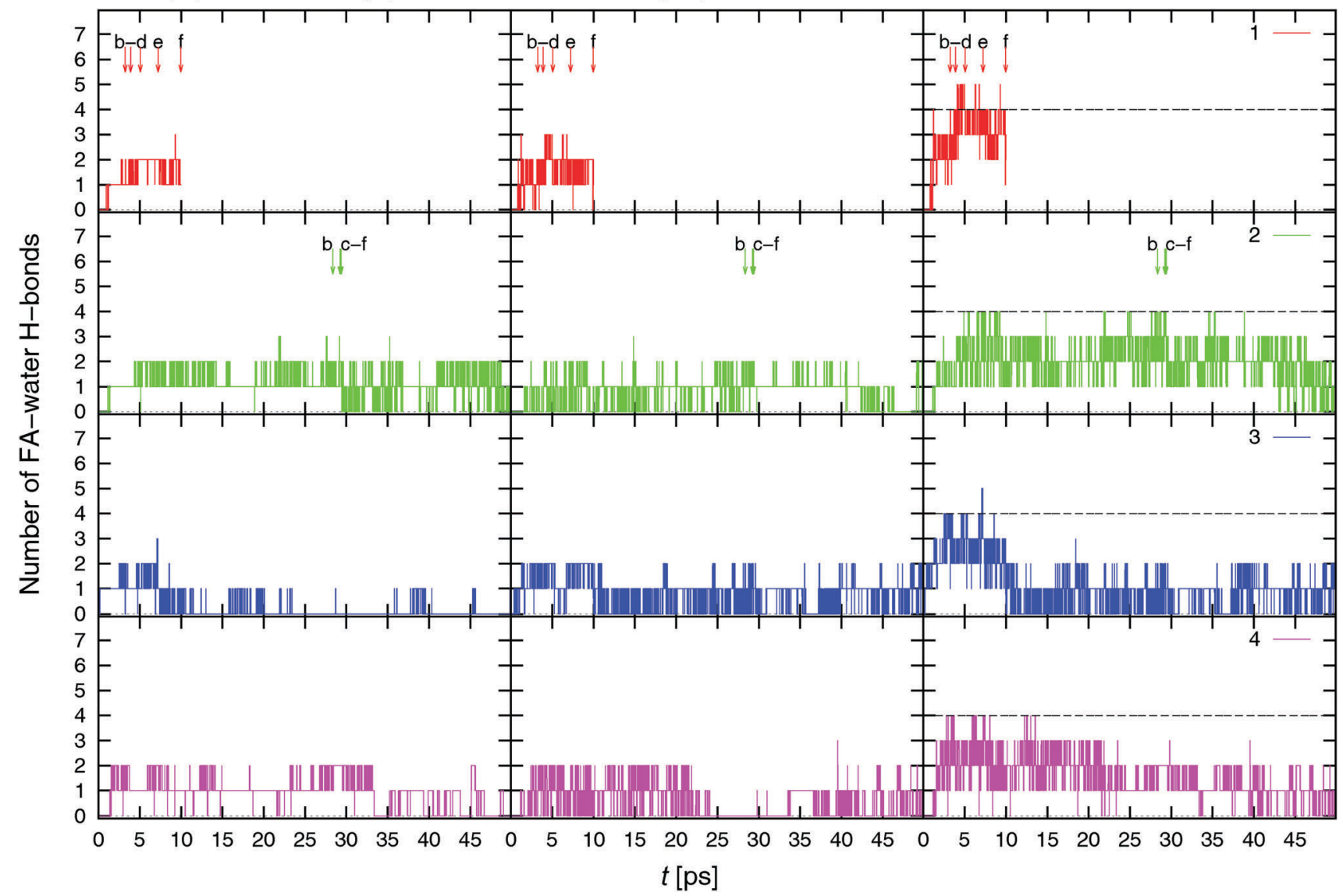

Fig. 9 The number of $\mathrm{H}$-bonds that FA forms with water molecules is shown for four selected collision trajectories. The rows, top to bottom, show collision trajectories 1-4. Left to right, the column in each row shows the number of FA-water $\mathrm{H}$-bonds involving the FA hydroxyl group (-OH), the FA carbonyl group $(-\mathrm{C}=\mathrm{O})$, and the total number of $\mathrm{H}$-bonds, respectively. The dotted black line at $4 \mathrm{H}$-bonds in the panels in the rightmost column marks the approximate $\mathrm{H}$-bonding threshold for FA ionization (3-4 H-bonds). The arrows labeled b-f refer to the snapshots shown in Fig. 5 (trajectory 1 ) and 6 (trajectory 2).

Table 1 Average number of $\mathrm{H}$-bonds that each oxygen atom of formic acid (neutral, unless otherwise indicated) participates in and the total number of $\mathrm{H}$-bonds in the four aqueous environments as obtained from equilibrium simulations. ${ }^{a}$ Literature values are also presented for formic acid and the formate ion in bulk water. Hydration numbers are shown in parentheses

\begin{tabular}{|c|c|c|c|c|}
\hline & Ref. & $(\mathrm{O}) \mathrm{H} \cdots \mathrm{Ow}$ and $\mathrm{O}(\mathrm{H}) \cdots \mathrm{Hw}$ & $(\mathrm{C}=) \mathrm{O} \cdots \mathrm{Hw}$ & Total \\
\hline Center of slab & This work & 1.8 & 1.0 & $2.8 \pm 0.9$ \\
\hline Subsurface of slab & This work & 1.2 & 1.8 & $3.0 \pm 0.5$ \\
\hline Surface of slab & This work & 1.9 & 1.1 & $3.0 \pm 0.6$ \\
\hline Bulk water & 81 (Theory: DFT) & & & $3.0^{b}$ \\
\hline Bulk water (formate ion) & 83 and 84 (Experiment) & & & $\left(<4.6-4.9^{d}\right)$ \\
\hline Bulk water (formate ion) & 85 (Experiment: IR) & & & $>3^{e}$ \\
\hline
\end{tabular}

${ }^{a}$ The data were obtained by averaging the data shown in Fig. S5 of the ESI. ${ }^{b}$ Estimated from the hydration number of 3.1 obtained from the O-Hw radial distribution function reported in ref. 81. ${ }^{c}$ This is the hydration number obtained from the O-Ow radial distribution function. Such a hydration number will always be an overestimate of the number of $\mathrm{H}$-bonds. ${ }^{d}$ Total hydration numbers of formate as estimated from X-ray diffraction and Raman/ATR-IR experiments, extrapolated to infinite dilution. ${ }^{e}$ Experiments and theory.

Fig. 11 compares the results of the simulations of FA in bulk liquid water (black line) and in the center of the slab (blue line). The two curves are in reasonable agreement given that our slab is relatively thin. Next, the results for FA sampled at two depths in the interfacial region, the subsurface $\left(z=z_{\mathrm{GDS}}-1 \AA\right)$ and surface $\left(z=z_{\mathrm{GDS}}+1 \AA\right.$ ) are shown (see also Fig. 2). 

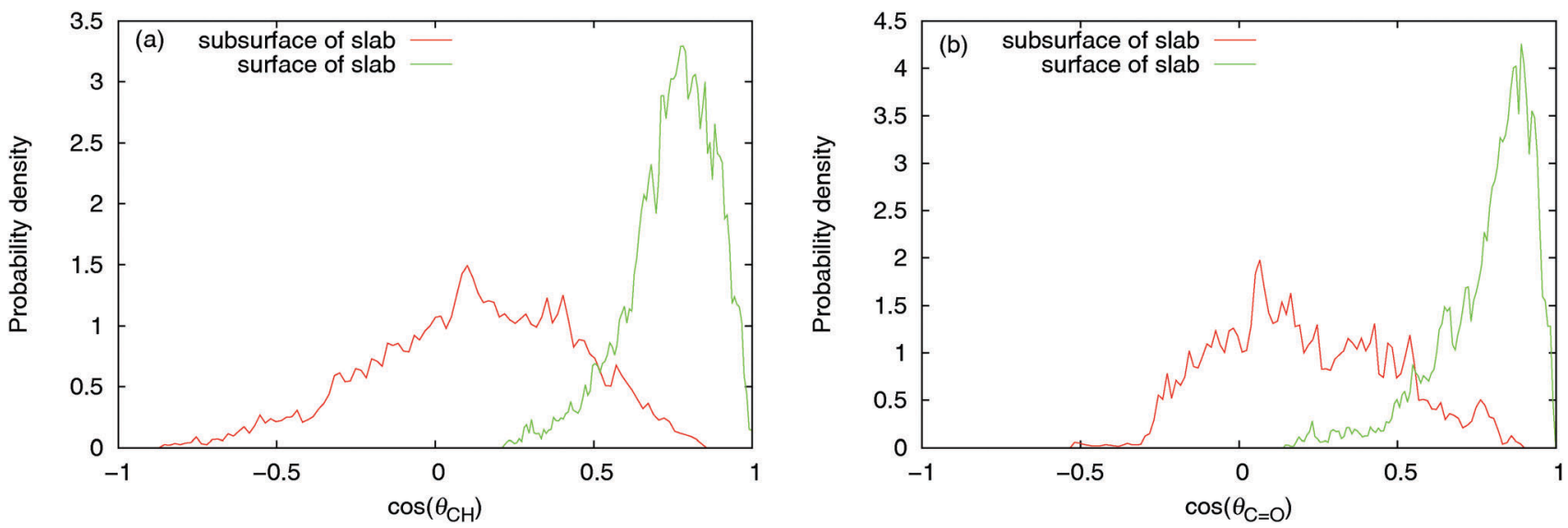

Fig. 10 Distributions of the cosines of two orientation angles of neutral trans-FA at two depths in the interfacial region obtained from equilibrium simulations: (a) $\cos \theta_{\mathrm{CH}}$ and (b) $\cos \theta_{\mathrm{C}=\mathrm{O}}$. The extracted average values and standard deviations of the angles and the comparison to the experimental values reported in ref. 32 are: (a) $\theta_{\mathrm{CH}}^{\text {surface }}=42 \pm 12^{\circ}\left(\theta_{\mathrm{CH}}^{\text {exp }}=27-43^{\circ}\right), \theta_{\mathrm{CH}}^{\text {subsurface }}=83 \pm 18^{\circ}$; and (b) $\theta_{\mathrm{C}}^{\text {surface }}=38 \pm 14^{\circ}\left(\theta_{\mathrm{C}}^{\text {exp }} \mathrm{O}=42-57^{\circ}\right), \theta_{\mathrm{C}=0}^{\text {subsurface }}=78 \pm 16^{\circ}$. Also see the ESI $\dagger$ for the $\cos \theta_{n}$ and $\cos \theta_{\mathrm{CO}}$ distributions.

Their deprotonation barriers are similar, and the two curves have been averaged together to obtain the average behavior at the interface.

Our barrier to FA deprotonation in liquid water is somewhat low at $14.8 \mathrm{~kJ} \mathrm{~mol}^{-1}$, compared to barriers of about $16.7-18.0 \mathrm{~kJ} \mathrm{~mol}^{-1}$ found by Lee $e t a l .{ }^{38}$ The underestimation of the barrier in the bulk liquid is due to an underestimation of free energy which we attribute mainly to our use of a relatively small DZVP basis set, since our preliminary calculations using the larger MOLOPT-DZVP-SR basis set ${ }^{86}$ indicate that the barrier becomes about $2.1 \mathrm{~kJ} \mathrm{~mol}^{-1}$ deeper in the bulk liquid. Here, we are ignoring nuclear quantum effects and any possible deficiencies of the DFT BLYP-D2 description of the electronic structure, which would also account for differences with respect to, for

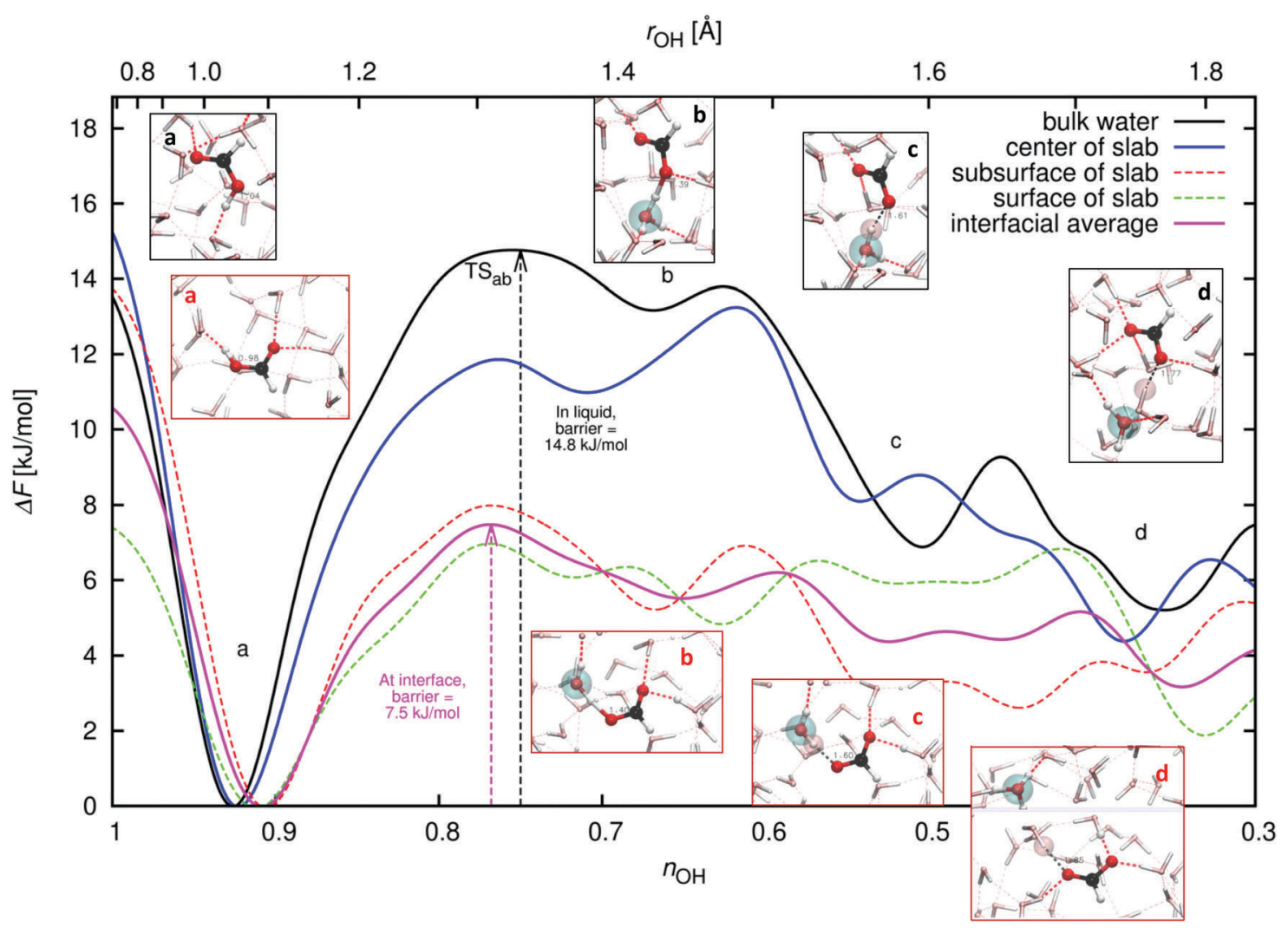

Fig. 11 Results of the well-tempered metadynamics simulations of formic acid deprotonation in liquid water and at the water surface. The free energy curves $\Delta F=\Delta F\left(n_{\mathrm{OH}}\right)$ and barriers are shown for trans-formic acid dissociation in various environments. The insets show typical snapshots at the indicated state points in the bulk (black borders) and at the subsurface (red borders). See the text for details. 
example, experimental and calculated $\mathrm{p} K_{\mathrm{a}} \mathrm{s}$. We do not attempt to compute $\mathrm{p} K_{\mathrm{a}} \mathrm{s}$ since that would require converging states $\mathrm{c}$ (CIP) or d (SSIP). The uncertainty in the interfacial curve, if considered as the deviation between the subsurface and surface curves becomes large in the $\mathrm{c}$ and $\mathrm{d}$ regions.

Considering the interfacial curve, we obtain a barrier of $7.5 \mathrm{~kJ} \mathrm{~mol}^{-1}$, a reduction of nearly $50 \%$ relative to the barrier in the bulk of $14.8 \mathrm{~kJ} / \mathrm{mol}$. Our results imply that formic acid deprotonates faster at the air-water interface than when fully solvated in water. This observation may be reconciled with the lack of observed deprotonated FA at the air-water interface in the XPS experiments by Brown et al. ${ }^{29,30}$ and in the VSFG experiments by Johnson et al. ${ }^{32,33}$ by noting that deprotonation is a relatively rare event.

\section{Concluding remarks and atmospheric chemistry implications}

We have employed ab initio molecular dynamics with dispersioncorrected density functional theory to study an atmospherically prevalent medium-strength acid, formic acid (FA), at the air-water interface. Our scattering simulations confirm that FA is strongly surface active, and remains adsorbed even in our longest simulations of 50 ps. Deprotonation happens in only about $4 \%$ of our simulations. When it occurs, it initiates PT whose duration ranges from fractions of a picosecond to a few picoseconds, indicative of concerted and sequential proton transfer mechanisms, respectively. PT terminates with the reformation of neutral FA, which may however have undergone a trans to cis conformational change. With respect to our total trajectory time of collisions of about 590 ps, we observed the existence of deprotonated FA in only about $1 \%$ of the time (about $6 \mathrm{ps}$ in the two trajectories) before the neutral FA was reformed by various mechanisms. With regard to neutral FA, our simulations show an agreement between the orientation of FA at the top of the air-water interface and those obtained in the VSFG experiments by Johnson et al. ${ }^{32,33}$ We may partially reconcile the lack of observed deprotonated formic acid in these VSFG experiments by appealing to the rapid homogenization of formic acid orientation with depth, making VSFG experiments less sensitive to the depth where deprotonation appears to be more favored.

Our metadynamics simulations comparing the deprotonation barriers of FA at the air-water interface and in the bulk indicate that it has a smaller barrier to dissociation at the interface which implies that FA deprotonates faster at the interface. A detailed examination of the equilibrium solvation and $\mathrm{H}$-bonding state of FA in interfacial compared to bulk solvation is inconclusive since approximately the same average number of $\mathrm{H}$-bonds are obtained. Thus, the faster deprotonation at the interface may be partially due to the faster water re-orientational and H-bond dynamics at the interface or it may be due to the effects in the second solvation shell. Regardless of the cause, since all carboxylic acids have a strong surface preference, the faster deprotonation at the interface implies that these acids, particularly formic and acetic acids which can have significant concentrations especially in polluted atmospheres and in rain and cloud water, may contribute more strongly to atmospheric acidity than previously believed, and produce ionic products, which even if short-lived, can enhance pickup and reactions with adsorbed species such as amines.

The behavior of FA at the air-water interface is contrary to that of many strong acids. Baer et al. ${ }^{1}$ have shown that $\mathrm{HCl}$ has a similarly small barrier to deprotonation at the interface as in the bulk (but the CIP is only stable at the interface), while $\mathrm{HNO}_{3}$ has an exceptionally large barrier at the surface due to insufficient solvation and exceptional stability of neutral $\mathrm{HNO}_{3}$. Thus at the surface, $\mathrm{HNO}_{3}$, depending also on its concentration, can be effectively a weak acid and may be observed to be neutral. ${ }^{2,3}$ Therefore, the simple impression of the higher concentration of under-coordinated water molecules at the interface leading to enhanced reactivity need not always be true.

It should be of considerable interest to extend this work to other carboxylic acids $\mathrm{RCOOH}$, many of which are known to participate in atmospheric systems and processes, e.g., in secondary organic aerosols. A point that merits attention is how does the organic residue $\mathrm{R}$ affect the ionization mechanism and dynamics. What is well-known is that with increasing size of the (relatively hydrophobic) R group, the molecule's solubility (i.e., absorption into the water bulk) decreases. This can be quantified by, for example, the Henry's Law constant, which for acetic acid is about $40 \%$ less $^{41}$ than that of formic acid. Also, given the amphiphilic nature of carboxylic acids, the orientational preference observed with formic acid, with the $\mathrm{R}$ group oriented along the surface normal, is maintained. However, more work is merited on the deprotonation propensity, dynamics, and mechanisms of other $\mathrm{RCOOH}$, at the interface, in comparison to their fully solvated behavior. Biological chemistry and astrochemistry/prebiotic chemistry are other disciplines where the interactions of such acids in an aqueous environment are potentially of much interest. ${ }^{87-89}$

\section{Acknowledgements}

We are grateful to Finland's Center for Scientific Computing (CSC) for providing computational resources. RBG thanks the Israel Science Foundation (ISF Grant 172/12), the US National Science Foundation (NSF Grants CHE 0909227 and 1443140), and AirUCI for partial support of this project. GMN thanks the National Science Foundation (CHE-1152737) for financial support. LH thanks the Academy of Finland (grant numbers 257479 and 294752) for financial support. GM acknowledges discussions with Lauri Partanen and thanks him for reading and commenting on the manuscript.

\section{References}

1 M. D. Baer, D. J. Tobias and C. J. Mundy, J. Phys. Chem. C, 2014, 118, 29412-29420.

2 M. C. Kido Soule, P. G. Blower and G. L. Richmond, J. Phys. Chem. A, 2007, 111, 3349-3357. 
3 T. Lewis, B. Winter, A. C. Stern, M. D. Baer, C. J. Mundy, D. J. Tobias and J. C. Hemminger, J. Phys. Chem. C, 2011, 115, 21183-21190.

4 T. Lewis, B. Winter, A. C. Stern, M. D. Baer, C. J. Mundy, D. J. Tobias and J. C. Hemminger, J. Phys. Chem. B, 2011, 115, 9445-9451.

5 E. S. Shamay, V. Buch, M. Parrinello and G. L. Richmond, J. Am. Chem. Soc., 2007, 129, 12910-12911.

6 S. Wang, R. Bianco and J. T. Hynes, J. Phys. Chem. A, 2009, 113, 1295-1307.

7 D. Ardura and D. J. Donaldson, Phys. Chem. Chem. Phys., 2009, 11, 857-863.

8 C. D. Wick, J. Phys. Chem. A, 2013, 117, 12459-12467.

9 G. Murdachaew, M.-P. Gaigeot, L. Halonen and R. B. Gerber, J. Phys. Chem. Lett., 2013, 4, 3500-3507.

10 G. Murdachaew, M.-P. Gaigeot, L. Halonen and R. B. Gerber, Phys. Chem. Chem. Phys., 2014, 16, 22287-22298.

11 S. Riikonen, P. Parkkinen, L. Halonen and R. B. Gerber, J. Phys. Chem. Lett., 2013, 4, 1850-1855.

12 S. Riikonen, P. Parkkinen, L. Halonen and R. B. Gerber, J. Phys. Chem. A, 2014, 118, 5029-5037.

13 R. B. Gerber, M. E. Varner, A. D. Hammerich, S. Riikonen, G. Murdachaew, D. Shemesh and B. J. Finlayson-Pitts, Acc. Chem. Res., 2015, 48, 399-406.

14 Y. R. Shen and V. Ostroverkhov, Chem. Rev., 2006, 106, 1140-1154.

15 G. M. Nathanson, Annu. Rev. Phys. Chem., 2004, 55, 231-255.

16 I. Chorny, I. Benjamin and G. M. Nathanson, J. Phys. Chem. B, 2004, 108, 995-1002.

17 S. M. Brastad and G. M. Nathanson, Phys. Chem. Chem. Phys., 2011, 13, 8284-8295.

18 L. P. Dempsey, S. M. Brastad and G. M. Nathanson, J. Phys. Chem. Lett., 2011, 2, 622-627.

19 J. A. Faust and G. M. Nathanson, Chem. Soc. Rev., 2016, 45, 3609-3620.

20 J. A. Faust, T. B. Sobyra and G. M. Nathanson, J. Phys. Chem. Lett., 2016, 7, 730-735.

21 L. Partanen, G. Murdachaew, R. B. Gerber and L. Halonen, Phys. Chem. Chem. Phys., 2016, 18, 13432-13442.

22 B. J. Finlayson-Pitts and J. N. Pitts, Chemistry of the Upper and Lower Atmosphere: Theory, Experiments, and Applications, Academic Press, San Diego, CA, USA, 2000.

23 R. P. Wayne, Chemistry of Atmospheres: An Introduction to the Chemistry of the Atmospheres of Earth, the Planets, and their Satellites, Oxford University Press, Oxford, 2000.

24 A. Chebbi and P. Carlier, Atmos. Environ., 1996, 30, 4233-4249.

25 P. Khare, N. Kumar, K. M. Kumari and S. S. Srivastava, Rev. Geophys., 1999, 37, 227-248.

26 S. Schobesberger, F. D. Lopez-Hilfiker, D. Taipale, D. B. Millet, E. L. D'Ambro, P. Rantala, I. Mammarella, P. Zhou, G. M. Wolfe, B. H. Lee, M. Boy and J. A. Thornton, Geophys. Res. Lett., 2016, 2016 GL069599.

27 J. Hedberg, J. Henriquez, S. Baldelli, C. M. Johnson and C. Leygraf, J. Phys. Chem. C, 2009, 113, 2088-2095.

28 S. Enthaler, J. von Langermann and T. Schmidt, Energy Environ. Sci., 2010, 3, 1207-1217.
29 M. A. Brown, F. Vila, M. Sterrer, S. Thürmer, B. Winter, M. Ammann, J. J. Rehr and J. A. van Bokhoven, J. Phys. Chem. Lett., 2012, 3, 1754-1759.

30 J. G. Pruyne, M.-T. Lee, C. Fábri, A. Beloqui Redondo, A. Kleibert, M. Ammann, M. A. Brown and M. J. Krisch, J. Phys. Chem. C, 2014, 118, 29350-29360.

31 N. Ottosson, E. Wernersson, J. Söderström, W. Pokapanich, S. Kaufmann, S. Svensson, I. Persson, G. öhrwall and O. Björneholm, Phys. Chem. Chem. Phys., 2011, 13, 12261-12267.

32 C. M. Johnson, E. Tyrode, A. Kumpulainen and C. Leygraf, J. Phys. Chem. C, 2009, 113, 13209-13218.

33 C. M. Johnson and S. Baldelli, Chem. Rev., 2014, 114, 8416-8446.

34 D. Priem, T.-K. Ha and A. Bauder, J. Chem. Phys., 2000, 113, 169-175.

35 S. Aloisio, P. E. Hintze and V. Vaida, J. Phys. Chem. A, 2002, 106, 363-370.

36 E. G. Goken and A. W. Castleman, J. Geophys. Res.: Atmos., 2010, 115, D16203.

37 E. G. Goken, K. L. Joshi, M. F. Russo, A. C. T. van Duin and A. W. Castleman, J. Phys. Chem. A, 2011, 115, 4657-4664.

38 J.-G. Lee, E. Asciutto, V. Babin, C. Sagui, T. Darden and C. Roland, J. Phys. Chem. B, 2006, 110, 2325-2331.

39 L. B. Pártay, P. Jedlovszky, P. N. M. Hoang, S. Picaud and M. Mezei, J. Phys. Chem. C, 2007, 111, 9407-9416.

40 P. Jedlovszky, G. Hantal, K. Neuróhr, S. Picaud, P. N. M. Hoang, P. v. Hessberg and J. N. Crowley, J. Phys. Chem. C, 2008, 112, 8976-8987.

41 B. J. Johnson, E. A. Betterton and D. Craig, J. Atmos. Chem., 1996, 24, 113-119.

42 J. Staudinger and P. V. Roberts, Chemosphere, 2001, 44, 561-576.

43 R. Sander, Atmos. Chem. Phys., 2015, 15, 4399-4981.

44 The CP2K developers group, http://www.cp2k.org/, 2000-2014.

45 A. D. Becke, Phys. Rev. A: At., Mol., Opt. Phys., 1988, 38, 3098-3100.

46 C. Lee, W. Yang and R. G. Parr, Phys. Rev. B: Condens. Matter Mater. Phys., 1988, 37, 785-789.

47 S. Grimme, J. Comput. Chem., 2006, 27, 1787-1799.

48 S. Goedecker, M. Teter and J. Hutter, Phys. Rev. B: Condens. Matter Mater. Phys., 1996, 54, 1703-1710.

49 A. D. Hammerich and V. Buch, J. Phys. Chem. A, 2012, 116, 5637-5652.

50 A. D. Hammerich, B. J. Finlayson-Pitts and R. B. Gerber, J. Phys. Chem. Lett., 2012, 3, 3405-3410.

51 A. D. Hammerich, B. J. Finlayson-Pitts and R. B. Gerber, Phys. Chem. Chem. Phys., 2015, 17, 19360-19370.

52 I.-F. W. Kuo, C. J. Mundy, B. L. Eggimann, M. J. McGrath, J. I. Siepmann, B. Chen, J. Vieceli and D. J. Tobias, J. Phys. Chem. B, 2006, 110, 3738-3746.

53 G. Murdachaew, C. J. Mundy, G. K. Schenter, T. Laino and J. Hutter, J. Phys. Chem. A, 2011, 115, 6046-6053.

54 M. Pettersson, J. Lundell, L. Khriachtchev and M. Räsänen, J. Am. Chem. Soc., 1997, 119, 11715-11716.

55 K. Marushkevich, L. Khriachtchev and M. Räsänen, J. Phys. Chem. A, 2007, 111, 2040-2042. 
56 G. J. Martyna, M. L. Klein and M. Tuckerman, J. Chem. Phys., 1992, 97, 2635-2643.

57 D. J. Tobias, G. J. Martyna and M. L. Klein, J. Phys. Chem., 1993, 97, 12959-12966.

58 A. P. Willard and D. Chandler, J. Phys. Chem. B, 2010, 114, 1954-1958.

59 M. D. Baer, C. J. Mundy, M. J. McGrath, I.-F. W. Kuo, J. I. Siepmann and D. J. Tobias, J. Chem. Phys., 2011, 135, 124712.

60 A. Laio and M. Parrinello, Proc. Natl. Acad. Sci. U. S. A., 2002, 99, 12562-12566.

61 M. Iannuzzi, A. Laio and M. Parrinello, Phys. Rev. Lett., 2003, 90, 238302.

62 A. Barducci, G. Bussi and M. Parrinello, Phys. Rev. Lett., 2008, 100, 020603.

63 J. F. Dama, M. Parrinello and G. A. Voth, Phys. Rev. Lett., 2014, 112, 240602.

64 M. Sprik, Chem. Phys., 2000, 258, 139-150.

65 G. Murdachaew, M. E. Varner, L. F. Phillips, B. J. Finlayson-Pitts and R. B. Gerber, Phys. Chem. Chem. Phys., 2012, 15, 204-212.

66 G. M. Nathanson and T. B. Sobyra, et al., article in preparation.

67 A. Hassanali, F. Giberti, J. Cuny, T. D. Kühne and M. Parrinello, Proc. Natl. Acad. Sci. U. S. A., 2013, 110, 13723-13728.

68 M. J. Cox, R. L. A. Timmer, H. J. Bakker, S. Park and N. Agmon, J. Phys. Chem. A, 2009, 113, 6599-6606.

69 B. J. Siwick and H. J. Bakker, J. Am. Chem. Soc., 2007, 129, 13412-13420.

70 P. Maurer, V. Thomas and R. Iftimie, J. Chem. Phys., 2011, 134, 094505.

71 S. Miura, M. E. Tuckerman and M. L. Klein, J. Chem. Phys., 1998, 109, 5290-5299.
72 J. Kohanoff, S. Koval, D. A. Estrin, D. Laria and Y. Abashkin, J. Chem. Phys., 2000, 112, 9498-9508.

73 C. Drechsel-Grau and D. Marx, Phys. Rev. Lett., 2014, 112, 148302.

74 X. Meng, J. Guo, J. Peng, J. Chen, Z. Wang, J.-R. Shi, X.-Z. Li, E.-G. Wang and Y. Jiang, Nat. Phys., 2015, 11, 235-239.

75 C. Drechsel-Grau and D. Marx, Nat. Phys., 2015, 11, 216-218.

76 V. R. I. Kaila and G. Hummer, Phys. Chem. Chem. Phys., 2011, 13, 13207-13215.

77 D. Marx, ChemPhysChem, 2007, 8, 209-210.

78 N. Agmon, Chem. Phys. Lett., 1995, 244, 456-462.

79 D. Marx, M. E. Tuckerman, J. Hutter and M. Parrinello, Nature, 1999, 397, 601-604.

80 K. R. Leopold, Annu. Rev. Phys. Chem., 2011, 62, 327-349.

81 Q. Chen, Z. Liu and C. H. Wong, J. Theor. Comput. Chem., 2012, 11, 1019-1032.

82 K. Leung and S. B. Rempe, J. Am. Chem. Soc., 2004, 126, 344-351. 83 Y. Kameda, K. Fukuhara, K. Mochiduki, H. Naganuma, T. Usuki and O. Uemura, J. Non-Cryst. Solids, 2002, 312, 433.

84 Y. Kameda, T. Mori, T. Nishiyama, T. Usuki and O. Uemura, Bull. Chem. Soc. Jpn., 1996, 69, 1495-1504.

85 M. Smiechowski, E. Gojlo and J. Stangret, J. Phys. Chem. B, 2011, 115, 4834-4842.

86 J. VandeVondele and J. Hutter, J. Chem. Phys., 2007, 127, 114105.

87 T. C. Freitas, K. Coutinho, M. T. d. N. Varella, M. a. P. Lima, S. Canuto and M. H. F. Bettega, J. Chem. Phys., 2013, 138, 174307.

88 A. N. Borkar, M. K. Rout and R. V. Hosur, PLoS One, 2011, 6, e19830.

89 R. T. Garrod, S. L. W. Weaver and E. Herbst, Astrophys. J., 2008, 682, 283-302. 